\title{
HIERARCHICAL MATRIX APPROXIMATIONS OF HESSIANS ARISING IN INVERSE PROBLEMS GOVERNED BY PDES*
}

\author{
ILONA AMBARTSUMYAN ${ }^{\dagger}$, WAJIH BOUKARAM ${ }^{\ddagger}$, TAN BUI-THANH $^{\dagger}$, OMAR GHATTAS $^{\dagger}$, \\ DAVID KEYES $\ddagger$, GEORG STADLER ${ }^{\S}$, GEORGE TURKIYYAH ${ }^{\uparrow}$, AND STEFANO ZAMPINI ${ }^{\ddagger}$
}

\begin{abstract}
Hessian operators arising in inverse problems governed by partial differential equations (PDEs) play a critical role in delivering efficient, dimension-independent convergence for both Newton solution of deterministic inverse problems, as well as Markov chain Monte Carlo sampling of posteriors in the Bayesian setting. These methods require the ability to repeatedly perform such operations on the Hessian as multiplication with arbitrary vectors, solving linear systems, inversion, and (inverse) square root. Unfortunately, the Hessian is a (formally) dense, implicitly-defined operator that is intractable to form explicitly for practical inverse problems, requiring as many PDE solves as inversion parameters. Low rank approximations are effective when the data contain limited information about the parameters, but become prohibitive as the data become more informative. However, the Hessians for many inverse problems arising in practical applications can be well approximated by matrices that have hierarchically low rank structure. Hierarchical matrix representations promise to overcome the high complexity of dense representations and provide effective data structures and matrix operations that have only log-linear complexity. In this work, we describe algorithms for constructing and updating hierarchical matrix approximations of Hessians, and illustrate them on a number of representative inverse problems involving time-dependent diffusion, advection-dominated transport, frequency domain acoustic wave propagation, and low frequency Maxwell equations, demonstrating up to an order of magnitude speedup compared to globally low rank approximations.
\end{abstract}

Key words. Hessians, inverse problems, PDE-constrained optimization, Newton methods, hierarchical matrices, matrix compression, log-linear complexity, GPU, low rank updates, NewtonSchulz.

1. Introduction. The Hessian operator plays a central role in optimization of systems governed by partial differential equations (PDEs), also known as PDEconstrained optimization. While the approach proposed here applies more broadly to other PDE-constrained optimization problems including optimal control and optimal design, we will focus on an important class: inverse problems. The goal of an inverse problem is to infer model parameters, given observational data, a forward model or state equation (here in the form of PDEs) mapping parameters to observables, and any prior information on the parameters. Often the parameters represent infinitedimensional fields, such as heterogeneous coefficients (including material properties), distributed sources, initial or boundary conditions, or geometry. We focus here on this infinite dimensional setting, leading to large scale inverse problems after discretization.

The Hessian operator plays a critical role in inverse problems. For deterministic inverse problems, finding the parameters that best fit the data is typically formulated as a regularized nonlinear least squares optimization problem. Its objective function(al) consists of two terms: the data misfit, that is, the squared $\ell^{2}$-norm of

Funding: This work was supported by the King Abdullah University of Science and Technology (KAUST) Office of Sponsored Research (OSR) under Award No: OSR-2018-CARF-3666.

$\dagger$ Oden Institute for Computational Engineering and Sciences, The University of Texas at Austin. (ailona@austin.utexas.edu, tanbui@ices.utexas.edu,omar@ices.utexas.edu).

${ }^{\ddagger}$ Extreme Computing Research Center, King Abdullah University of Science and Technology. (wajihhalim.boukaram@kaust.edu.sa, stefano.zampini@kaust.edu.sa, david.keyes@kaust.edu.sa).

§Courant Institute of Mathematical Sciences, New York University. (stadler@cims.nyu.edu).

\Department of Computer Science, American University of Beirut, Lebanon. (gt02@aub.edu.lb). 
the difference between the output observables predicted by the PDE for given model parameters and the observed data; and a regularization term that ensures stability by penalizing unobserved features of the parameters, such as rough components. The Hessian is given by the second variation, with respect to model parameters, of this regularized data misfit. Minimizing the objective by Newton's method requires solution of a sequence of linear systems with the Hessian as its system matrix. Due to its affine invariance and resulting mesh-independent behavior (e.g., [40]), Newton's method is the gold standard for inverse problem solution, with demonstrated convergence independent of parameter dimension for large-scale, complex inverse problems (e.g., $[33,44])$. Efficient solution of the linear systems arising at each Newton iteration requires an effective preconditioner that is inexpensive to form and "invert." The challenge is that the discretized Hessian of the data misfit functional is formally a dense operator that cannot be formed explicitly, since each column requires the solution of a linearized state PDE. Instead, we can exploit the fact that for ill-posed inverse problems, the data misfit Hessian operator is compact (its eigenvalues accumulate at zero), and when the regularization operator takes the typical form of an elliptic differential operator, we can precondition by the inverse of the regularization to yield a preconditioned Hessian in the form of a compact perturbation of the identity. A conjugate gradient linear solver will converge in a number of iterations that depends on the number of distinct eigenvalues, which for regularization preconditioning is small when the compact part has eigenvalues that decay rapidly, so that it has small effective rank $r$. Since at each iteration, applying the Hessian to a vector requires the solution of a pair of linearized state/adjoint PDEs, $O(r)$ such pairs must be solved at each Newton iteration. As demonstrated in a number of geophysical inversion problems involving global seismology [25], ice sheet flow [44], atmospheric transport [34], poroelastic subsurface flow [41], and joint inversion [29], $r$ is small and dimension-independent, and such a preconditioning strategy is effective. However, as we shall see below, the central challenge is that $r$ grows as the data become more informative (a desirable situation for inverse problems), and regularization preconditioning becomes prohibitive. ${ }^{1}$

Another critical role of the Hessian is in Bayesian inversion, which provides a powerful and systematic framework for quantifying uncertainties in the solution of inverse problems. Given probability distributions that represent observational data and their associated uncertainties, the state PDE model and its associated uncertainty, and any prior knowledge on the model parameters, Bayesian solution of the inverse problem yields the posterior distribution, which characterizes the probability that any particular parameter field gave rise to the observed data. The Hessian in this setting is given by the second variation (with respect to model parameters) of the negative $\log$ of the posterior distribution. This is equivalent to the deterministic Hessian in the common setting of Gaussian additive noise and prior. For linear inverse problems, the posterior covariance is equal to the inverse of the Hessian. For moderately nonlinear inverse problems, the posterior can be approximated by the Laplace approximation, in which the posterior covariance is given by the inverse Hessian evaluated at the point that maximizes the posterior covariance (or minimizes the negative log posterior). Finally, for highly nonlinear inverse problems, where the Laplace approximation does not adequately capture the uncertainty in the inverse solution, Markov chain Monte

\footnotetext{
${ }^{1}$ The augmented Lagrangian KKT preconditioner [4] overcomes this difficulty and is insensitive to $r$, but since it involves the optimality system of the PDE-constrained optimization problem, is storage-prohibitive for large-scale time-dependent inverse problems.
} 
Carlo (MCMC) methods are used to sample the posterior, from which a sample posterior covariance can be constructed. In this case the Hessian plays an instrumental role in exploiting the geometry of the posterior to accelerate MCMC convergence $[48,30,14,26]$. In all three cases, what is needed is an accurate and efficient method to approximate the Hessian, its inverse, and their square roots.

The contemporary approach to approximation of Hessians of ill-posed inverse problems is to again exploit the compactness of the Hessian of the data misfit functional. Compactness can be theoretically proven in specific settings (e.g., for acoustic and electromagnetic inverse shape and medium scattering [24, 23, 22]), or else demonstrated numerically for an array of inverse problems [10, 48, 21, 25, 74, 41, 56, 44, 34]. This often allows one to make a low rank approximation of the data misfit component of the Hessian (preconditioned by the prior covariance, which plays the role of the inverse regularization operator), computed efficiently via matrix-free randomized SVD [39]. This entails $O(r)$ products of the prior-preconditioned data misfit Hessian matrix with random vectors, each of which as above requires a pair of linearized state/adjoint PDE solutions. The inverse Hessian and its square root are then computed at negligible additional cost using the resulting spectral decomposition along with the Sherman-Morrison-Woodbury formula $[25,56]$. When the inverse problem is highly ill-posed, i.e., when the data inform few components of the parameter field, $r$ is small. Moreover, the eigenfunctions corresponding to the dominant eigenvalues of the Hessian are often smooth (as a consequence of the data being unable to inform rough components of the parameter field), so that $r$ is independent of the mesh size and hence parameter dimension. In such cases, the low rank approximation is both efficient and scalable (with respect to parameter dimension).

In summary, to ensure efficiency of CG solution with regularization preconditioning as well as low rank approximation of the prior-preconditioned data misfit Hessian, it is critical that the effective rank $r$ of the Hessian remain small relative to the parameter dimension. However, while $r$ generally does not grow with increasing parameter dimension, it does grow as the data become more informative about the parameters. In fact, for a linear Bayesian inverse problem with additive Gaussian noise, the Kullback-Leibler divergence (or relative entropy) from posterior to prior measure - which quantifies the information gained from the data - can be shown to be equal to $\Sigma_{i} \log \left(\lambda_{i}+1\right)$, where $\lambda_{i}$ are the eigenvalues of the prior-preconditioned data misfit Hessian [1]. This sum can be truncated when $\lambda_{i} \ll 1$, so the dominant eigenvalues - and hence effective rank of this operator-are directly related to the information content of the data. The information gained from the data (and hence $r$ ) generally increases as the number of sources (or experiments) increases and the number of observations (or sensors or receivers) increases. The data also become more informative as the state PDEs become less dissipative (for example as flow or transport equations become more advection-dominated), or as they resolve finer scales (such as in wave propagation with increasing frequency). Finally, as the noise in the data decreases, the strength of the data misfit Hessian increases relative to the prior/regularization, again increasing the effective rank and hence informativeness of the data. In all such cases, both regularization preconditioning as well as low rank-based data misfit Hessian approximation become prohibitive, and there remains a critical need for more efficient Hessian approximation.

In this paper, we show for the first time the efficiency of hierarchical matrix representations [37] in representing the Hessian operator of various PDE-constrained problems, and consider these tunable-accuracy approximations to address the shortcomings of the globally low rank representations in the increasingly data-informed 
regime, as well as to provide log-linear time complexity algorithms for constructing robust approximations of the inverse of these operators. Hierarchical matrices exploit the fact that many of their off-diagonal blocks can be approximated to high accuracy with blocks of low rank, allowing substantial compression in the memory footprint relative to the dense representation. The blocks that may be so represented can be of different sizes offering a natural tree hierarchy for managing and operating on them, and resulting in matrices that can be stored and operated on in linear or log-linear space and time complexity as opposed to polynomial complexity of dense representations. Such matrix representations provide an algebra in which memory versus accuracy tradeoffs may be explicitly made to suit the needs of specific application contexts, a particularly useful feature for algorithms that can operate with coarser approximations to benefit from substantial speedup. Hierarchical matrices may be viewed as algebraic generalizations of fast multipole methods [65], allowing the fast performance of not only matrix-vector products, but also a full stack of linear algebra operations including generalizations of randomized algorithms for constructing matrix decompositions $[39,20]$.

With the emergence of GPUs and manycore architectures as key platforms for high performance scientific computing, efficient execution on these architectures has become a critical feature for algorithms that aim to be deployable in practice. With this in mind, the algorithms we present for operating on hierarchical representations of Hessians are developed to exploit the high throughput of these modern architectures through data parallel operations and careful orchestration of data movement to minimize latencies. Linear algebra operations such as matrix-vector products, low rank updates, and matrix inversion can be performed directly on the GPU resident compressed representations, allowing algorithms to benefit both from the reduced memory footprint with its resulting log-linear operation count as well as from the high flop rate of modern hardware architectures.

Besides low rank approximation, several methods for compact approximation of (data misfit) Hessians stemming from inverse problems have been developed recently. The pseudodifferential scaling method of $[51,52]$ exploits the pseudodifferential nature of the Hessian in seismic inverse problems, in particular that it is approximately diagonal in phase space and can thus be estimated by a single application to a vector. Also exploiting the pseudodifferential structure of seismic inversion Hessians is the matrix probing method of [31], which approximates the Hessian (and its inverse) with basis matrices stemming from the Hessian's symbol and find their coefficients by probing the Hessian in random directions. Recently, methods that exploit the local translation invariance of Hessians have been introduced $[74,3]$. The adaptive product-convolution approximation in particular is demonstrated to be robust to the Peclet number for advection-dominated transport and the frequency for an auxiliary operator that arises in connection with KKT preconditioning [3] for a wave inverse problem [2]. Here we focus on comparisons with low rank approximation, and defer comparison to these other methods in appropriate contexts for future work.

The rest of this paper is organized as follows. In section 2, we argue why Hessians of PDE-governed inverse problems are expected to be well approximated by hierarchical matrices. Section 3 introduces the $\mathcal{H}^{2}$ data structures used to store the hierarchical matrix as well as key operations on the representation, including matrix-vector multiplication, compression, low rank updates, and construction from randomized sampling. In section 4 we present numerical experiments to assess the effectiveness of the hierarchical matrix Hessian representations on inverse problems governed by diffusion, transport, and acoustic and electromagnetic wave propagation. 
The experiments demonstrate the superiority of hierarchical matrix approximations over a low rank approximation as the data become more informative, with speedups ranging from two to over an order of magnitude. Section 5 concludes the paper.

2. Why do Hessians Admit Hierarchical Low Rank Representations?

The Hessians that arise in PDE-governed inverse problems (whether deterministic or Bayesian) may be approximated to specified accuracy by highly compressible matrices, a property they inherit from the underlying PDE operators [12], often reinforced by sparse observation operators. As argued above, the global rank of these Hessians increases as the data become more informative, such that a low rank approximation may no longer be tenable. However, as we argue below, these Hessians typically have blocks that can be well approximated by low rank representations with bounded rank. These blocks are at different levels of granularity because of their different sizes, making hierarchical matrix representations an ideal vehicle for storing and computing with Hessians.

In this paper, we consider inverse problems governed by PDEs with distributed parameter fields to be inferred from data. After discretization over a $d$-dimensional domain $\Omega$ (which without loss of generality we assume is conducted by finite elements), we obtain an optimization problem of the form

$$
\underset{m}{\operatorname{minimize}} J(m):=F(u(m))+\alpha R(m)
$$

where the state variables $u(m) \in \mathbb{R}^{N}$ depend on the model parameters $m \in \mathbb{R}^{n}$ via solution of the discretized PDEs

$$
g(m, u):=K(m) u-f=0,
$$

with coefficient matrix $K \in \mathbb{R}^{N \times N}$ and source $f \in \mathbb{R}^{N}$. For simplicity, we confine our discussion to the case where the forward problem $g(m, u)=0$ represents elliptic PDEs that have arbitrary dependence on the parameter but are linear in the state. However, our methodology is more broadly applicable to parabolic and hyperbolic forward problems, as demonstrated in the applications in section 4, and to nonlinear forward problems. Multiple sources (or multiple experiments) may be used to generate data, in which case (2.2) is indexed by source $s$, generating the state $u^{s}$.

The data misfit term $F(u(m))$ measures the difference between recorded observations and the response of the model at receivers placed on the boundary or the interior of $\Omega$. It is often taken to be the sum of squares, over all sources and receivers, of $d_{r}^{s}$, the difference between the observed state at receiver $r$ due to source $s$, and the corresponding model response $u_{r}^{s}$,

$$
F:=\frac{1}{2} \sum_{s} \sum_{r}\left\|u_{r}^{s}-d_{r}^{s}\right\|^{2}
$$

although other measures of misfit may be sometimes preferable [64]. $R(m)$ is a regularization term expressing prior information on the parameter field (such as piecewise smoothness) and $\alpha$ is a scalar regularization parameter that attempts to annihilate components of $m$ that are uninferable from the data while preserving those that can be inferred. The regularization term is generally a local operator whose discretization is sparse and does not pose computational difficulties even at large scale. Thus in this section we focus on the treatment of the problematic $F$ term, whose evaluation requires solution of the forward PDEs for a given $m$ for each source $s$. 
We define $G \in \mathbb{R}^{N \times n}$ to be the partial derivative of the forward PDE residual with respect to the parameter field, i.e.,

$$
G:=\partial_{m} g=\partial_{m} K \times_{2} u,
$$

where $\partial_{m} K$ is a third order tensor of size $N \times N \times n$ and $\times_{2}$ is the 2-mode tensor vector product operation in the notation of [45]. Since $K$ is a discretization of a local PDE operator, it is representable as a sparse matrix. The parameter field is typically discretized on the same mesh as the state (perhaps at lower order), and thus the local operator $G$ is also sparse.

Unlike the local sparse matrices $K$ and $G$, the inverse operator $K^{-1}$ is a discretized, non-local solution operator, and is in general formally dense. It is however data-sparse, since many of its off-diagonal blocks can be represented to high accuracy by low rank approximations with bounded rank $k$, and these blocks occur at different levels of granularity $[12,16]$. For a block $t s$ of $K^{-1}$, with rows and columns corresponding to the index set $t$ and $s$ of clusters of nodes of a finite element mesh, the compressibility condition states that if the bounding boxes of these clusters are sufficiently far away from each other, then the local rank of the $t s$ block is essentially independent of the block size for Poisson-like operators. Such a condition is known as an admissibility condition, and it depends on the spatial distance between the clusters of $t$ and $s$, relative to their size.

Using the nested basis $\mathcal{H}^{2}$ representation [15], the total amount of storage needed to represent $K^{-1}$ to a given accuracy $\epsilon$ is only $O(N k)$, where the local block rank $k$ grows as $|\log \epsilon|^{d+1}$ for PDEs in $d$ spatial dimensions. Note that the local ranks of the blocks are independent of the global rank. Even for matrices that are of full rank $N$, the local ranks grow slowly, with only a logarithmic dependence on target approximation accuracy, as well as with a logarithmic dependence on high contrast coefficients [11]. High-frequency problems can be tackled effectively by using directional compression techniques, with asymptotic storage $O\left(N k^{2} \log N\right)$ [17].

The gradient of the data misfit term of the objective function may be written in terms of an adjoint variable $p \in \mathbb{R}^{N}$, defined via the adjoint equations [43]

$$
l(m, p):=K^{T}(m) p+\partial_{u} F=0 .
$$

The gradient of $F$ is then given by

$$
\nabla_{m} F:=\left(\partial_{m} K \times_{2} u\right)^{T} p=-G^{T} K^{-T} \partial_{u} F .
$$

We denote by $L \in \mathbb{R}^{N \times n}$ the partial derivative of the residual of the adjoint equations (2.3) with respect to $m$,

$$
L:=\partial_{m} l=\partial_{m} K^{T} \times_{2} p,
$$

which is a local operator and has a sparsity pattern similar to that of $G$. Taking the derivative of (2.4) with respect to $m$, we obtain an expression for the Hessian matrix in terms of the state and adjoint variables $u$ and $p$, and the local operators $G$ and $L$,

$$
\nabla_{m m}^{2} F=G^{T} K^{-T} \partial_{u u}^{2} F K^{-1} G-G^{T} K^{-T} L-L^{T} K^{-1} G+\left(\partial_{m m}^{2} K \times_{2} u\right) \times_{1} p .
$$

The first term of the latter expression represents the positive semi-definite GaussNewton part of the Hessian, involving the application of the local operator $G$ and its adjoint from the left and right to the triple product $K^{-T}\left(\partial_{u u}^{2} F\right) K^{-1}$. The next two terms involve the application of the sparse Jacobians of the state and adjoint 
equations, from the left and from the right, to $K^{-1}$ and its adjoint $K^{-T}$. The last term involves a sparse, fourth-order tensor multiplied in 1-mode and 2-mode by the adjoint and state variables.

Equation (2.5) shows why it is prohibitive to form the Hessian operator explicitly: forming $K^{-1} G$ (and similar products) requires a number of forward PDE solves equal to the number of parameters multiplied by the number of sources. On the other hand, (2.5) also shows that products of the Hessian with arbitrary vectors can be carried out at the cost of one forward solve (with $K$ ) and one adjoint solve (with $K^{T}$ ). Note also that (unless the forward problem is linear in $m$ ), in general $u, p, K, G$, and $L$ all depend on $m$, so the Hessian varies over parameter space. However the argument below is not predicated on where in parameter space the Hessian is evaluated.

In the form given in (2.5), we can see why the Hessian of (2.1) exhibits a hierarchical low rank structure. We first consider the Gauss-Newton term. The observation operator $\partial_{u u}^{2} F$ is positive semi-definite, and in particular is block diagonal for the standard weighted least squares objective (where the blocks correspond to the support of the receiver operators). When the number of observations in $F$ is small, it could have relatively small global rank as well. A general result concerning the structure of the product of hierarchical matrices [35, Theorem 2.24] shows that the triple product $K^{-T} \partial_{u u}^{2} F K^{-1}$, even when $\partial_{u u}^{2} F$ is full rank, may be represented as a hierarchical matrix, although the local ranks may grow as a result. By the same argument, the right and left multiplications of the latter triple product by the sparse matrix $G$ and its transpose also produce a hierarchical matrix with bounded local ranks. For favorable sparsity structures in $G$, the resulting local ranks may even decrease. This is due to the fact that a block in the product contains contributions from a bounded number of block product pairs, where most of the operands involved are zero and contribute no data to the target block. The local ranks of the Gauss-Newton Hessian may therefore end up being smaller than the local ranks of the solution operator. The second and third terms of the full Hessian expression given in (2.5) also have the form of a hierarchical matrix multiplied on both sides by sparse matrices, and therefore result in hierarchical matrices with small local ranks. Finally, the last term of the Hessian involves the sparse fourth order tensor. The sum of all four terms may of course increase the local ranks slightly, but the resulting Hessian will still retain a hierarchical structure.

3. Hierarchical Matrix Construction of Dense Hessians. In this section we describe the hierarchical Hessian representation that results in linear space complexity, and show how to perform linear algebra operations on the Hessian directly in its compressed representation, including matrix-vector products and norm computations, in near linear time complexity. We also describe a randomized procedure for constructing hierarchical Hessian approximations to a desired accuracy $\epsilon$.

3.1. Structure and representation. Various representations for hierarchical matrices have been proposed in the past and may be roughly classified along two characteristics as shown in Table 3.1. One relates to the structure of the block partitioning of the matrix, and the other relates to the format of the representation and how the low rank data is stored. In the simplest representations, such as $\mathcal{H}_{p}$ [37] or HODLR [5] (see also [66, 67]), a weak-admissibility condition is used for partitioning, so that all the off-diagonal blocks touch the main diagonal, and each block at level $l$ of the hierarchy defined by the row and column index sets $t$ and $s$ respectively has its own low rank representation $A_{t s}^{l}=U_{t s}^{l} V_{t s}^{l^{T}}$. The HSS representation [63] improves the asymptotic complexity by using nested bases while still keeping the simple par- 
titioning. In nested basis representation, the column and row bases are no longer specific to a given block but shared with all blocks in the $t$ block row and $s$ block column, i.e. $A_{t s}^{l}=U_{t}^{l} S_{t s} V_{s}^{l^{T}}$. The bases $U_{t}$ and $V_{s}$ are not stored explicitly, but are computed recursively on demand from their "children," i.e., block rows and columns that are subsets of $t$ and $s$. Bases are only stored explicitly at the lowest level, and only small transfer matrices are needed to compute coarser levels bases, resulting in the asymptotically optimal $O(k n)$ storage, where $k$ is the maximum rank among $A_{t s}^{l}$ blocks.

\begin{tabular}{|l||c|c|}
\hline & Flat bases & Nested bases \\
\hline \hline Weak admissibility partitioning & HODLR $[5], \mathcal{H}_{p}[37]$ & HSS $[63]$ \\
\hline Strong admissibility partitioning & $\mathcal{H}[37]$ & $\mathcal{H}^{2}[15], \mathcal{D} \mathcal{H}^{2}[17]$ \\
\hline
\end{tabular}

Table 3.1: Classification of hierarchical matrix representations.

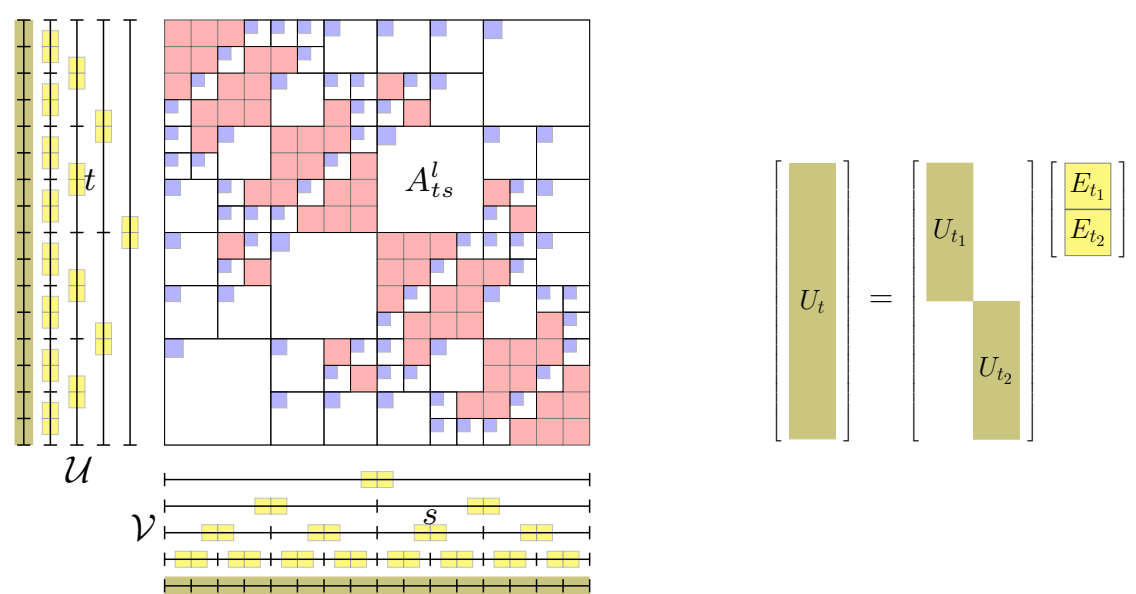

Fig. 3.1: $O(n)$ hierarchical representation of $A$ : matrix partitioning and nested bases. Low rank representation of the blocks is of the form $A_{t s}^{l}=U_{t}^{l} S_{t s}^{l} V_{s}^{l^{T}}$, with $U_{t}^{l}$ computed from the bases of its children $U_{t 1}^{l-1}$ and $U_{t 2}^{l-1}$ using small transfer matrices $E^{l-1}$ stored in a column basis tree $\mathcal{U}$. Per-block low rank data $S_{t s}$ are stored as small $k \times k$ blocks in a matrix tree $\mathcal{S}$.

The primary drawback of the weak admissibility partitioning is that the ranks of the off-diagonal blocks grow too large even when only a moderate accuracy is requested for many problems of interest, and in particular for three-dimensional problems. This undesirable growth in rank demands a more refined matrix blocking, tuned to the actual geometric discretization of the problem, to be used for the partitioning of the hierarchical matrix. In this blocking, identified as strong admissibility partitioning in the bottom row of Table 3.1, only those matrix blocks $t s$ whose $t$ and $s$ index sets define clusters that are sufficiently far away from each other will admit a low rank representation. This allows more refinements to take place on large blocks of the weak partitioning block structure. In this work, we use the $\mathcal{H}^{2}$ representation [15]; for a schematic representation of the storage format, see Figure 3.1. A general partitioning of the matrix is allowed, as prescribed by an application-dependent admissibility con- 
dition, quantifying the relative distance between clusters. Every admissible low rank block is represented by a triple product $U_{t} S_{t s} V_{s}^{T}$ : all the needed information for generating the $U_{t}$ columns bases is stored in the leaves of the $\mathcal{U}$ tree. For non-symmetric matrices, a separate row basis tree $\mathcal{V}$ is also needed. Blocks that do not admit a low rank representation are stored as dense blocks, and are limited to the leaf level of the matrix blocks. An informal notation for a hierarchical matrix $A$ is then

$$
A_{\mathcal{H}^{2}}=D+\mathcal{U} \cdot \mathcal{S} \cdot \mathcal{V}^{T}
$$

where $D$ is a block sparse matrix, and the tree triplet $\{\mathcal{U}, \mathcal{S}, \mathcal{V}\}$ provides the data for the low rank blocks at multiple levels of granularity. Hierarchical matrices may hence be viewed as generalizations of matrix decompositions of the form "diagonal plus low rank" or "sparse plus low rank" that have been used with success in PDE-governed inverse problems [25].

3.2. Fast operations on hierarchical matrices. The power of hierarchical matrices is not only a result of their optimal storage but of the fact that we can also perform general linear algebra operations directly in the compressed representation in linear or log-linear time complexity.

Matrix-vector multiplication, for example, can be done in four stages:

$$
\left.A_{\mathcal{H}^{2}} x=D x+\mathcal{U} \cdot \mathcal{S} \cdot \mathcal{V}^{T} \cdot x=D x+\mathcal{U} \cdot\left(\mathcal{S} \cdot\left(\mathcal{V}^{T} \cdot x\right)\right)\right)
$$

The part involving dense matrix blocks may be done via the usual block sparse multiplication separately from the low rank part. The latter is done by first applying the row basis tree $\mathcal{V}^{T}$ to $x$ and involves an upsweep in the $\mathcal{V}$ tree, with multiplication by the explicit basis at the leaves and the transfer matrices up the tree. This is followed by the application of $\mathcal{S}$ to this product for all blocks at all levels. Finally, the application of $\mathcal{U}$ on this intermediate result is done via a downsweep through the tree using the transfer matrices and the explicit bases at the leaves. This is the key idea of fast multipole methods and a similar operations count shows that the complexity is $O(k n)$. In addition, there is much concurrency in all stages and overlap between the stages can be exploited for efficient execution on GPUs [19].

Recompression is a basic operation on hierarchical matrices that we rely on for efficiently building and updating them. Consider for example the task of adding a low rank update to $A_{\mathcal{H}^{2}}$,

$$
\tilde{A}_{\mathcal{H}^{2}}=A_{\mathcal{H}^{2}}+X Y^{T}
$$

where $X$ and $Y$ are global low rank matrices of size $n \times k^{\prime}$. This update will evidently affect all blocks of $A_{\mathcal{H}^{2}}$ at all levels in the hierarchy, since the triple product form of a given block in the updated matrix may be expressed as:

$$
\tilde{A}_{t s}^{l}=\left[\begin{array}{ll}
U_{t} & X_{t}
\end{array}\right]\left[\begin{array}{cc}
S_{t s} & 0 \\
0 & I
\end{array}\right]\left[\begin{array}{c}
V_{s}^{T} \\
Y_{s}^{T}
\end{array}\right]
$$

where $X_{t}$ and $Y_{s}$ are restrictions of $X$ and $Y$ to the index sets $t$ and $s$, respectively. The local ranks of the matrix blocks have now increased to $k+k^{\prime}$. The leaves of the basis tree $\mathcal{U}$ and its transfer matrices have also increased by $k^{\prime}$. Recompression is the problem of finding new bases $\overline{\mathcal{U}}$ and $\overline{\mathcal{V}}$ with rank $\bar{k}<k+k^{\prime}$ and projecting the matrix $\tilde{A}$ on these bases, to obtain the compressed representation $\bar{A}$ such that the error introduced is below the target threshold to which $A$ itself is represented, $\|\tilde{A}-\bar{A}\| \leq \epsilon$. 


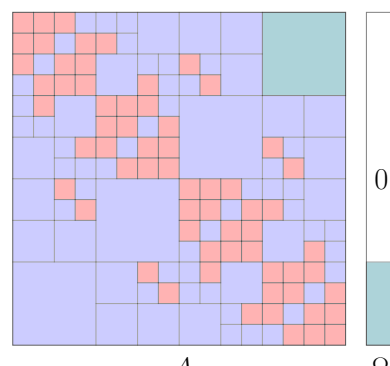

A
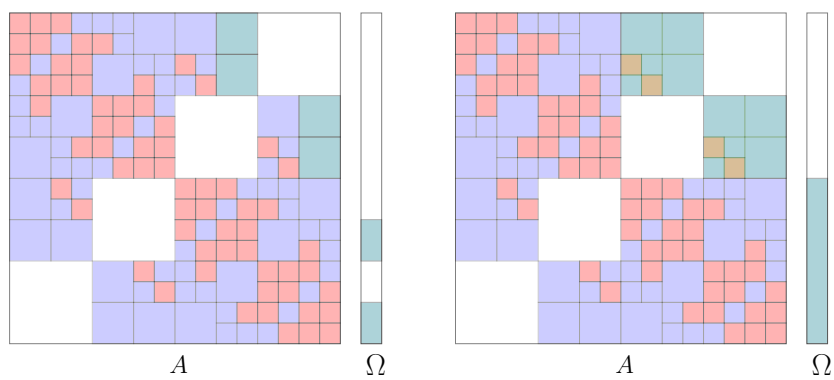

Fig. 3.2: Block sampling patterns. Sampled blocks need not correspond to the block structure of the hierarchical Hessian being constructed as illustrated in the figure on the right.

The new bases may be expressed as $U_{t} R_{t}$ where $R_{t}$ is the result of computing a $Q R$ factorization of the block row defined by $t$, and it can be computed quite efficiently via a downsweep pass through the basis trees performing $Q R$ factorizations only on small dense blocks. $U_{t} R_{t}$ is then truncated to the target accuracy via an SVD or randomized SVD via an upsweep pass through the basis trees performing SVD operations only on small dense blocks. All recompression computations may be done in log-linear time complexity, and there is substantial parallelism in all of the tree sweep operations, which can be exploited in manycore architectures. For further algorithmic and implementation details on the recompression procedure, see [19].

Matrix norms are also required by various operations, and they can be efficiently obtained via fast matrix-vector products or by accessing the low rank blocks. The exact Frobenius norm of a hierarchical matrix with an orthogonal basis can be efficiently evaluated in $O(k n)$ by simply taking the sum of the squares of the Frobenius norms of the coupling matrices of all blocks, $\|A\|_{F}^{2}=\sum\left\|A_{t s}\right\|_{F}^{2}=\sum\left\|U_{t} S_{t s} V_{s}^{T}\right\|_{F}^{2}=\sum\left\|S_{t s}\right\|_{F}^{2}$. On the other hand, $p$-norms can be approximated by a sequence of matrix-vector products using for example an iterative $p$-norm power method [42]. While the 1-norm and $\infty$-norm only require a very small number of iterations to converge, the 2-norm can require a relatively large number of iterations. In this case, we terminate the iterations when the norm value has converged to only 2 significant digits. In practice this is a good enough threshold for the relative norm estimation used in this work.

3.3. Construction from Hessian-vector products. Here we describe how to construct the hierarchical Hessians ab initio. In [66, 67], a method for constructing a hierarchical representation is presented that relies on having $O(1)$ access to individual matrix entries. In [49], construction of an HSS approximation is presented that also requires $O(1)$ access to entries. Unfortunately, in the case of the Hessians originating from PDE-constrained problems, access to individual entries in constant time is not generally possible, as these operators are only available in the form of matrix-vector products. For example, given an arbitrary vector in $\mathbb{R}^{n}$, the Hessian matrix-vector product for inverse problems governed by (time-dependent) PDEs is computed by solving the (forward-in-time) forward and (backward-in-time) adjoint equations as well as second order incremental state-like and adjoint-like PDEs, and by assembling the resulting product from spatio-(temporal) integrals involving the state, adjoint, incremental state, and incremental adjoint solution variables [43]. Examples of this procedure for inverse problems governed by several different classes of PDEs are described in section 4.

In order to generalize the successful randomized methods of dense matrix factor- 
izations [39] to hierarchical matrices, a number of procedures [46, 50, 20] have been proposed. As with dense matrices, these procedures sample the matrix via products with random vectors. The generalization to hierarchical matrices proceeds by levels, from coarsest to finest, and uses patterned random vectors chosen to sample particular blocks of the matrix. Figure 3.2 illustrates how this local sampling works; a column space for the low rank representation of the top right block (in the left figure) is generated by matrix-vector products with random columns $\Omega$ having nonzero values corresponding to the block being sampled. The resulting product has in its top block row, $(A \Omega)_{1}$, the desired column space, and the rest of the product may be discarded. A $Q R$ factorization of $(A \Omega)_{1}$ gives the orthogonal columns basis $U$ and the product $V=A^{T}\left[\begin{array}{ll}0 & U^{T}\end{array}\right]^{T}$ gives the row basis for the block and its representation $U V^{T}$. This process is repeated until the approximation of the sampled block satisfies an approximation threshold [20]. Similar low rank representation for the remaining blocks at the same level are performed, and they are then added as local low rank updates to the hierarchical matrix being constructed.

The blocks of the next level in the hierarchy are processed in a similar fashion but by sampling a matrix from which the higher level blocks previously sampled have been removed, as illustrated in the middle panel of Figure 3.2. Note that a single sampling matrix can be used to generate low rank representations of multiple blocks simultaneously as long as other blocks do not interfere with the sampling. Once the low rank block representations are generated, they are added as local low rank updates and the matrix is recompressed. We note here that we may sample larger blocks than the destination matrix has, as shown in the right panel of Figure 3.2 and our current implementation uses a weak admissibility sampling structure. Low rank representations of these sampling blocks are generated similarly and then added to multiple blocks of the destination matrix via local low rank updates. If the ranks of these blocks are too large there may be an advantage in sampling with larger blocks as the matrix is peeled in fewer steps, albeit with every step requiring more matrix-vector products.

For every level $l$ in the matrix, we therefore need $C_{l} k$ Hessian vector products, where $C_{l}$ is a constant that depends on how many blocks can be sampled simultaneously at level $l$, and $k$ is a representative local rank. The total number of samples is then $C k \log n$. The constant $C$ is small if we choose large sampling blocks (which will likely have larger local ranks $k$ ), but it is larger if we choose sampling strategies with smaller blocks. Therefore tuning the granularity of the sampling, which affects $C$ and $k$, may be needed, and it depends on the structure of the Hessian and on the relative costs of the matrix-vector products. It is also worth noting here that on modern hardware architectures, doing $C_{l} k$ matrix-vector products, i.e., solving multiple state and adjoint PDEs simultaneously, may not be much more expensive that solving for a single column, as the computations involved are usually memory bound and a multiple right-hand side approach will substantially increase the arithmetic intensity and the resulting throughput of the algorithm. As a result, operations count may not be the best complexity metric to evaluate performance.

The above procedure may be combined with global low rank approximations and may be accelerated if a Hessian approximation is available, e.g., for a different parameter from a previous iteration. For example, in situations where the data available for inversion is limited, the Hessian of the data misfit has a small finite dimensional range space, and the global Hessian rank may be small relative to its size. In these cases, an initial global small-rank approximation $B B^{T}$ of the misfit Hessian may be computed by the usual dense randomized sampling methods. If the global rank approximation 
does not reach the desired target accuracy, the hierarchical construction can be done on the residual $\left(H-B B^{T}\right)$ matrix, and $B B^{T}$ may then be added as a low rank update to it. This will likely require a smaller total number of matrix-vector products for the sampling relative to directly building the hierarchical Hessian.

A similar procedure can be used if a Hessian approximation is available, e.g., from a previous optimization iteration for a different parameter. Denoting the Hessian approximation by $\tilde{H}$, one can construct a hiearchical approximation (or a global low rank approximation) for the difference $H-\tilde{H}$. Alternatively, provided $\tilde{H}$ is invertible and we have an inverse square root $\tilde{H}^{-1 / 2}$ available, one could compute an approximation to $\left(\tilde{H}^{-1 / 2} H \tilde{H}^{-1 / 2}-I\right)$. In the same spirit, regularization matrices, which are generally sparse but have full rank, may be readily added via local low rank updates after the hierarchical misfit Hessian is constructed to obtain an explicit full Hessian representation that can then be efficiciently operated on. We discuss iterative inversion methods, which may also be adapted to square root and inverse square root computations, in Appendix A. Clearly, other linear algebraic methods for these computations are also possible once the compressed matrix representation of the Hessian is available.

4. Illustrative Applications. In this section, we discuss the effectiveness of the hierarchical representation of Hessians for several inverse problems, governed by a time-dependent diffusion equation in one spatial dimension (subsection 4.1), and in two space dimensions by a steady-state advection-diffusion equation (subsection 4.2), a frequency-domain wave equation (subsection 4.3), and a time-dependent low-frequency Maxwell equation (subsection 4.4). The last two examples are prototypes for geophysical inverse problems with seismic and controlled-source electromagnetic (CSEM) modalities, respectively.

The problems we consider are infinite-dimensional inverse problems, i.e., we infer parameter fields. We use a deterministic inverse problem approach, i.e., we use an optimization formulation and employ regularization to cope with the inherent ill-posedness. Note that under certain assumptions, the resulting PDE-constrained optimization problem can also be interpreted in a Bayesian context, in which case the minimizer corresponds to the maximum a posteriori parameter estimate. Upon discretization with finite elements (details on the discretization is given for each problem individually), these problems results in large-scale optimization problems, which we solve using an inexact Newton-conjugate gradient descent method with linesearch $[32,53]$. This requires availability of gradients and of Hessian-vector products, which are computed using adjoint methods as summarized in each example separately below. Similar methods are common in PDE-constrained optimization, [43, 18], and have successfully been used in large-scale optimization formulations of inverse problems, e.g., [25, 33, 74, 44, 41]. Here, we mainly focus on the approximations of the Hessian, which are always evaluated at the parameter field found as the solution of the optimization problem. In particular, we study the cost in terms of the number of Hessian applications (in PDE-constrained optimization this is usually the dominating cost) required for the construction of H-matrix approximations, and compare it with the cost of global low rank approaches. We also study the influence of the number of observations and properties of the governing PDEs on the compressibility of Hessian matrices.

4.1. Density inversion in 1D time-dependent diffusion equation. We consider a one-dimensional domain where we seek to invert for a spatially-varying porosity coefficient $\rho(x)$ in the governing diffusion equation $\rho(x) \partial_{t} u-\partial_{x}^{2} u=w(x, t)$. 
We use 3 sources and 8 receivers placed as shown in Figure 4.1. The point sources produce a Ricker wavelet time history input $W$ and the signals are recorded at the receivers until they have effectively dissipated at a final time $T>0$.
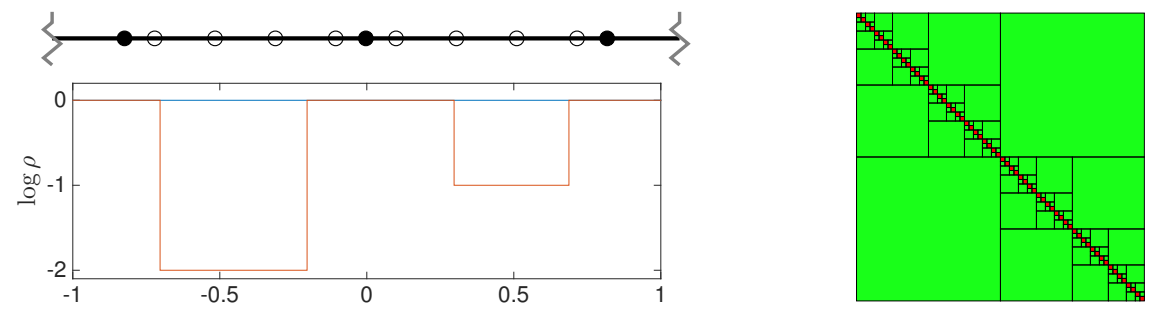

Fig. 4.1: (left) Sources $(\bullet)$, receivers (०), and target profile; (right) Hessian structure.

The medium to be recovered is in the interval $-1 \leq x \leq 1$ and has sharp discontinuities (see Figure 4.1), prompting the use of a (smoothed) total-variation regularization such that the optimization formulation is:

$$
\underset{\rho:[-1,1] \rightarrow \mathbb{R}}{\operatorname{minimize}} J(\rho):=\frac{1}{2} \sum_{s} \sum_{r} \int_{0}^{T}\left(u_{s}\left(x_{r}, t\right)-d_{r}(t)\right)^{2} d t+\alpha \int_{-1}^{1} \sqrt{\left|d_{x} \rho\right|^{2}+\beta} d x
$$

where for all sources $s, u_{s}(x, t)$ is the solution to

$$
\begin{aligned}
\rho(x) \partial_{t} u_{s}-\partial_{x}^{2} u_{s} & =\delta\left(x-x_{s}\right) W\left(t-t_{0}\right), & & |x|<\infty, 0 \leq t \leq T, \\
u_{s}( \pm \infty, t) & =0, & & (\text { Dirichlet BC) } \\
u_{s}(x, 0) & =0 . & & (\text { IC) }
\end{aligned}
$$

Here, the first term of the objective is the least squares time misfit summed over all sources and receivers. The second term is a total-variation regularization functional that allows sharp discontinuities to be recovered, where $\alpha>0$ is the regularization weight and a small $\beta>0$ ensures that the objective is differentiable.

For the computations below, $\rho(x)$ is discretized in $[-1,1]$ using $n$ linear elements. A finite element discretization of the governing equation with the same mesh is used in $[-1,1]$ and extended outside with constant $\rho=1$ to apply the homogeneous Dirichlet boundary conditions at a sufficiently far distance. An implicit second-oder time integration scheme is used for solving the semi-discrete equations.

The computation of the gradient may be done at the cost of two PDE solutions, a forward and an adjoint. The continuous form of the gradient of $J$, its functional Fréchet derivative, is

$$
\frac{\delta J}{\delta \rho}(x)=\sum_{s} \int_{0}^{T} \partial_{t} u_{s}(x, t) p_{s}(x, t) d t
$$

where, for each source $s, u_{s}$ is the solution of the state equation (4.2), and $p_{s}$ the solution of the corresponding adjoing equation that must be solved backwards-in time:

$$
\begin{gathered}
\text { (adjoint) } \quad-\rho(x) \partial_{t} p_{s}-\partial_{x}^{2} p_{s}=-\sum_{r} \delta\left(x-x_{r}\right)\left(u_{s}\left(x_{r}, t\right)-d_{r}(t)\right) \\
q( \pm \infty, t)=0 ; \quad q(x, T)=0 .
\end{gathered}
$$

To compute a hierarchical representation of the Hessian, we need the ability to compute products of the Hessian of the misfit term with vectors generated as 
described in section 3. This may be done at the cost of solving, for each source $s$, two additional forward-like and adjoint-like PDEs. The continuous form of the Hessianvector product, i.e., the product of the Fréchet Hessian with a model perturbation $\nu(x)$ is:

$$
\frac{\delta^{2} J}{\delta \rho^{2}} \nu=\sum_{s}\left(\int_{0}^{T} \partial_{t} u(x, t) q(x, t) d t+\int_{0}^{T} \partial_{t} v(x, t) p(x, t) d t\right)+\frac{\delta^{2} R}{\delta \rho^{2}} \nu,
$$

where the last term denotes the Hessian of the TV regularization $R$, and $v_{s}$ and $q_{s}$ are the solutions of the following incremental or second order forward and adjoint equations:

$$
\begin{gathered}
\rho(x) \partial_{t} v_{s}-\partial_{x}^{2} v_{s}=-\nu(x) \partial_{t} u_{s}(x, t), \\
v_{s}( \pm \infty, t)=0 ; \quad v_{s}(x, 0)=0, \\
\left(2^{\text {nd }} \text { order forward }\right) \\
\left.q_{s} \text { order adjoint }\right) \\
-\rho(x) \partial_{t} q_{s}-\partial_{x}^{2} q_{s}=-\sum_{r} \delta\left(x-x_{r}\right) v_{s}\left(x_{r}, t\right)-\nu(x) \partial_{t} p_{s}(x, t), \\
q_{s}( \pm \infty, t)=0 ; \quad q_{s}(x, T)=0 .
\end{gathered}
$$

The structure of the $\mathcal{H}$-representation of the Hessian is depicted in the right panel of Figure 4.1. We used a weak admissibility partitioning of the hierarchical matrix as appropriate for a one-dimensional spatial domain. There are $2^{l}$ symmetric blocks pairs at level $l$, each of size $n / 2^{l}$. For this example of size $n=2048$, we use 6 levels in the hierarchy, stopping the matrix refinement at blocks of size $m=32$. We construct the Hessian to a relative accuracy of about $10^{-6}$ in the 2-norm. The local ranks of the resulting matrix are shown in the left panel of Figure 4.2. The ranks plotted are the maximum local ranks for all off-diagonal blocks for every level. Even though the Hessian is full rank because of the regularization term, we note that its local ranks are small relative compared to its size even when a relatively tight tolerance of $10^{-6}$ is used in the construction.
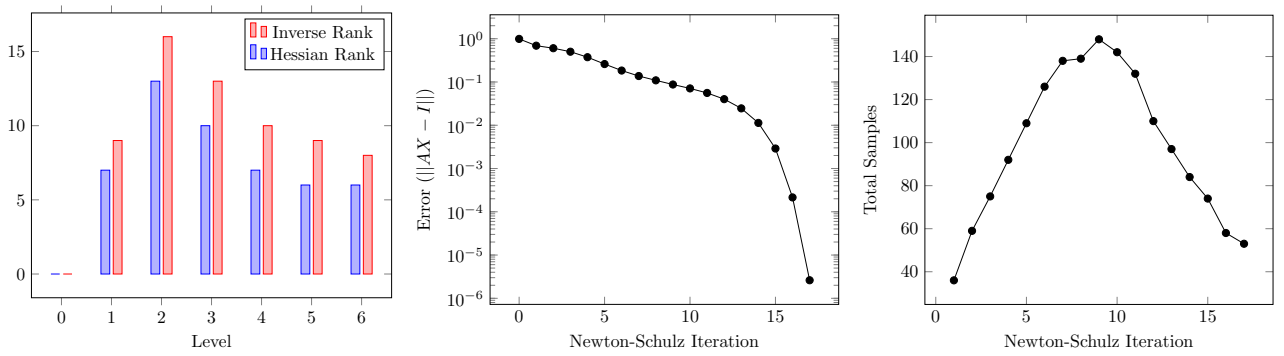

Fig. 4.2: (left) Local ranks of Hessian and its inverse. (middle) Convergence history of NS. (right) Number of samples needed to construct the matrix over the iterations.

Given this explicit representation of the Hessian, we can invert it to produce an explicit inverse. The convergence behavior of Newton Schulz starting from a scaled identity initial iterate to a relative accuracy of $\epsilon=10^{-6}$ is shown in Figure 4.2. The number of samples needed to construct the hierarchical approximation of the iterates is shown as well. In this simple $1 \mathrm{D}$ setting, we used a static threshold and a constant accuracy in the iterates. The increase in the required samples, and the corresponding local ranks, of the intermediate iterates is noteworthy. The left panel of Figure 4.2 shows the local ranks of the inverse along with the Hessian local ranks for comparison. There is little change in the local ranks in the inversion operation. 
4.2. Source inversion in stationary advection-diffusion. We consider a linear source inversion problem, i.e., we infer the right hand side source $m(x)$ in an advection-diffusion-reaction equation from point observations of its solution on a domain $\Omega \subset \mathbb{R}^{2}$. This amounts to the following optimization problem:

$$
\underset{m: \Omega \rightarrow \mathbb{R}}{\operatorname{minimize}} J(m):=\frac{1}{2 \sigma^{2}} \sum_{r}\left(u\left(x_{r}\right)-d_{r}\right)^{2}+R(m),
$$

where $u$ is the solution of

$$
\begin{aligned}
-\operatorname{div}(\kappa \nabla u)+\mathbf{v} \cdot \nabla u+c u & =m & & \text { in } \Omega, \\
u & =0 & & \text { on } \Gamma_{D}, \\
\kappa \frac{\partial u}{\partial n} & =0 & & \text { on } \Gamma_{N} .
\end{aligned}
$$

Here, the measurements $d_{r}$ are assumed to contain additive independent and identically distributed Gaussian noise with variance $\sigma$, and $R(m)$ is a regularization term. The coefficients $\kappa, \mathbf{v}$ and $c$ represent the diffusivity, the advective velocity and the reaction constant, respectively, and $\Gamma_{D}, \Gamma_{N} \subset \partial \Omega$ is a splitting of the boundary $\partial \Omega$, where we impose Dirichlet or Neumann boundary conditions, respectively.
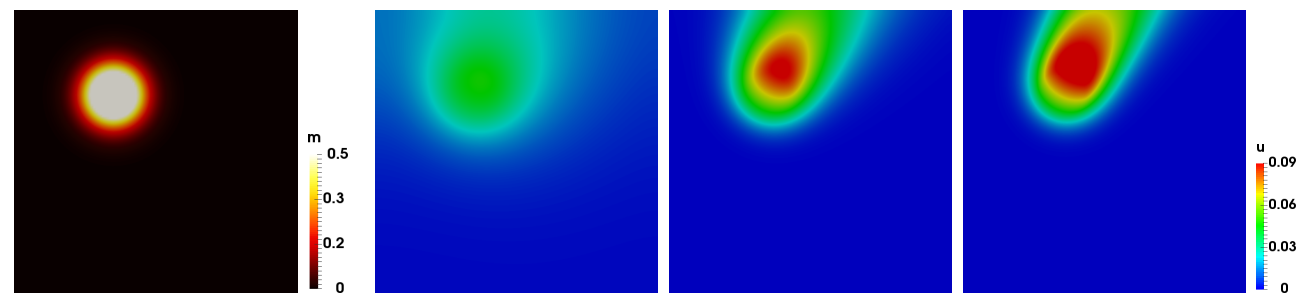

Fig. 4.3: Dependence of the solution for different diffusion parameters $\kappa$ : Shown on the left is the source $m$ entering on the right hand side of (4.6). The right three plots show the solutions of the state equation for $\kappa=10^{-1}, 10^{-2}, 10^{-3}$ (from left to right). Depending on $\kappa$, the problem is diffusion- or advection-dominated.

For our study of the Hessian, we use $\Omega=[0,1]^{2}$, the physical parameters $c=0.5$ and $\mathbf{v}=\left(x_{1}, x_{2}\right)^{T}$, and a noise level of $1 \%$. To study the influence of the diffusion and the number of observations, we use different values of $\kappa \in\left\{10^{-3}, 10^{-2}, 10^{-1}\right\}$ and different numbers of observation points, $N_{o b s} \in\{250, \ldots, 16000\}$.

We discretize $\Omega$ using triangles based on a uniform grid of size $128 \times 128$, and use linear continuous finite elements to discretize the parameter $m$. The presence of strong advection in the problem mandates the use of stabilization for the state and adjoint variables. We employ the first-order SIPG scheme [7, 57] with upwinding [8] for the forward and adjoint problems. Our implementation is based on FEniCS [47] and hIPPYlib, an open-source library providing scalable adjoint-based algorithms for PDE-based inverse problems [62]. The true source and the state solutions corresponding to the different values of the diffusion coefficient are shown in Figure 4.3.

In our study of the compressibility of the Hessian and the associated computational cost, we only consider the misfit Hessian, i.e., neglect the Hessian of the regularization term. This allows us to compare $\mathcal{H}$-matrix approaches with the more commonly used global low rank approach, which would suffer from inclusion of the Hessian of the regularization, which typically has full rank. Note that since this is 

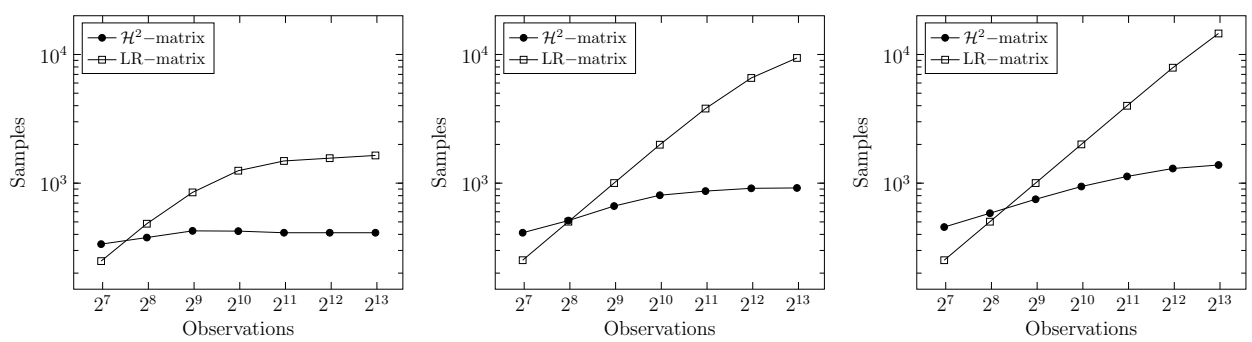

Fig. 4.4: The $y$-axis shows the number of Hessian-vectors products (samples) needed for constructing hierarchically and globally low rank approximations of the misfit Hessian to an accuracy of $\epsilon=10^{-4}$ for increasing number of observations plotted on the $x$-axis. Shows are results for $\kappa=10^{-1}$ (left), $10^{-2}$ (middle), and $10^{-3}$ (right).

a linear inverse problem, this misfit Hessian does not depend on the parameter $m$. Figure 4.4 shows the number of Hessian-vector products needed for constructing the misfit Hessian to a relative spectral accuracy of $10^{-4}$ for three different values of the diffusion coefficient $\kappa$ and an increasing number of observations. For small numbers of observations, the misfit Hessian has a fast decaying spectrum and a globally low rank representation can be achieved at a lower cost than with the hierarchical approach, measured in the number of Hessian-vector products. However, as more observations are incorporated, the Hessian applications required for the global low rank approximation grows rapidly. In contrast, the cost for the hierarchical compression only increases mildly with the number of observations as it is insensitive to the global rank. We also observe that larger diffusion coefficients $\kappa$ limit the increase of computational cost for increasing number of observations for both, the hierarchical and the global low rank approximations. This is a consequence of the fact that diffusion limits the amount of fine-scale information that can be recovered in the inversion, and thus more observations do not have a significant effect on the rank of the misfit Hessian.
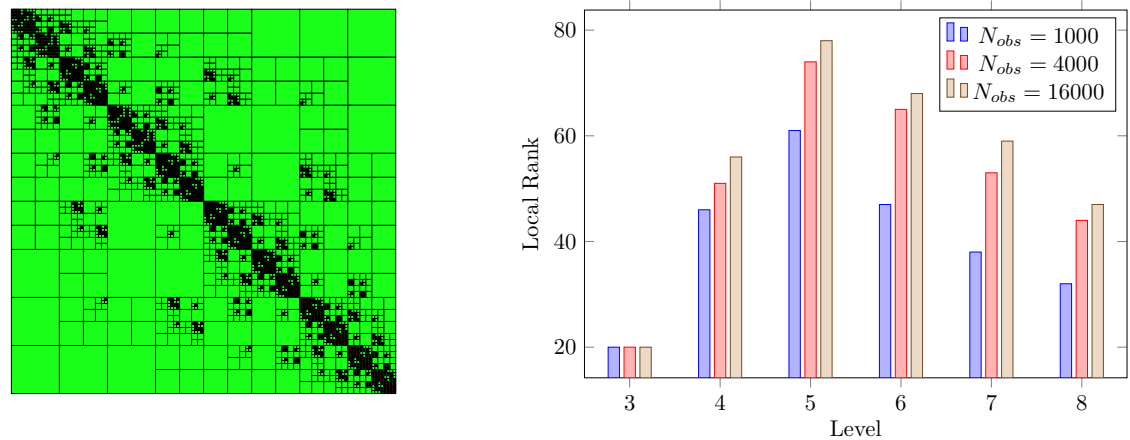

Fig. 4.5: 8-level structure of Hessian (left) and corresponding maximum local ranks per level for different number of observations and $\kappa=10^{-3}$ (right).

The relative insensitivity of the number of samples to the data dimension is reflected in the fact that the blocks of the resulting $\mathcal{H}^{2}$ have small local ranks that do not grow much even as the global rank of the matrix increases. The left panel of Figure 4.5 shows the structure of the constructed matrix which uses a node ordering of the mesh obtained from a KD-tree binary space partitioning method, with leaf 
clusters of size 64 , and an admissibility condition that resulted in the refined matrix partitioning shown. The right panel of Figure 4.5 shows how the block ranks grow much slower than the global Hessian rank as the data dimension of the problem grows.

4.3. Frequency-domain wave equation inversion. We consider the inverse acoustic wave propagation problem in frequency domain governed by the Helmholtz equation in a bounded domain $\Omega \subset \mathbb{R}^{2}$ :

$$
\Delta u+\omega^{2} q u=f, \quad \text { in } \Omega,
$$

where $u$ is the time-harmonic pressure, $f$ is the source and $w$ is the angular frequency. One corresponding inverse problem is to infer for the squared slowness, $q(x)=1 / c^{2}(x)$, where $c(x)$ is the speed of sound. We assume that the observations are given as the frequency-weighted normal derivative of the time-harmonic pressure at the receivers, located at all discretization points $x_{r}$ on the top boundary of the domain.

Assuming homogeneous Dirichlet boundary conditions and introducing the linear observation operator $B$ defined by $B(u)\left(x_{r}\right)=\omega^{2} \nabla u\left(x_{r}\right) \cdot n$, the deterministic inverse problem with an appropriate regularization term $R(q)$ can written as follows:

$$
\underset{q: \Omega \rightarrow \mathbb{R}}{\operatorname{minimize}} J(q):=\frac{1}{2} \sum_{r}\left(B(u)\left(x_{r}\right)-d_{r}\right)^{2}+R(q),
$$

where $u$ solves (4.7) with $u=0$ on $\partial \Omega$.

In order to be able to compare with global low rank methods, we do not include the regularization term in the gradient and Hessian expressions below, nor include them in our compression experiments. The continuous gradient of the misfit objective $F$ at a parameter $q_{0}$ is given by:

$$
\frac{\partial F}{\partial q}\left(q_{0}\right)=\omega^{2} q_{0} u_{0} v
$$

where $u_{0}$ is the solution of the state problem (4.7) with slowness $q_{0}$ and $v$ solves the adjoint problem:

$$
\Delta v+\omega^{2} q_{0} v=-\sum_{r}\left(B(u)\left(x_{r}\right)-d_{r}\right) \text { in } \Omega, \quad v=0 \text { on } \partial \Omega .
$$

In our numerical experiments, we use the Gauss-Newton approximation $H^{\mathrm{GN}}$ of the Hessian, which neglects terms involving the adjoint variable. This approximation is commonly used in practice since, differently from the Hessian, it is guaranteed to be positive semidefinite. In matrix form, it is given by

$$
H^{\mathrm{GN}}=C^{T} A^{-T} B^{T} B A^{-1} C,
$$

where $A$ stems for the discretization of the state operator, and $C$ from the discrete representation of the partial derivative of the PDE with respect to the parameter.

Our numerical test is set up using the 2D acoustic Marmousi model [61], a benchmark model for seismic inversion. The initial "hard" model is smoothed using a Gaussian filter to produce the result shown in Figure 4.6, which we use to generate synthetic observations. We consider a triangulation of the the rectangular domain $\Omega=[0,9192] \times[0,2904] \mathrm{m}^{2}$ in order to obtain about ten grid points per wave length. The state, adjoint and parameter variables are discretized using continuous piecewise 


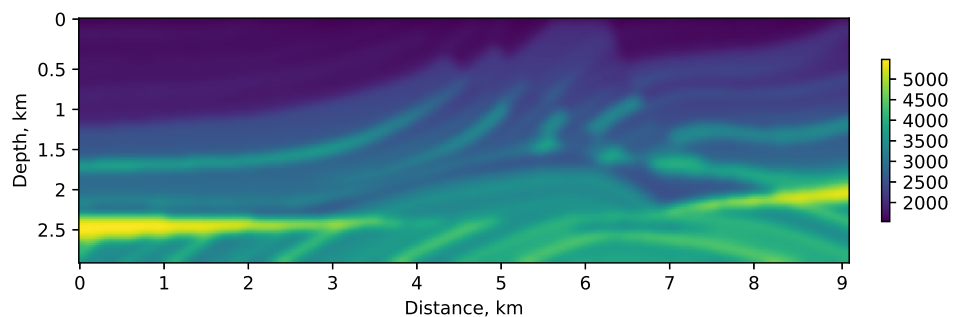

Fig. 4.6: Smoothed Marmousi model used to generate synthetic observations.

linear Lagrangian finite elements. Since we use the same finite element basis for the state $u$ and the parameter $q$, the $i j$ entry of $\mathrm{C}$ reads as

$$
C\left(u_{0}, q_{0}\right)_{i j}=\omega^{2} \int_{\Omega} \phi_{j} u_{0} \phi_{i} d x
$$

The right-hand side term in (4.7) is given as a sum of sources, located at a depth of $10 \mathrm{~m}$, spaced evenly every $12.5 \mathrm{~m}$. We further incorporate Dirichlet boundary condition along the top boundary of the domain and PML absorbing boundary conditions [13] on the other three boundaries. As in the previous problem, our implementation uses FEniCS and hiPPYlib [47, 62].

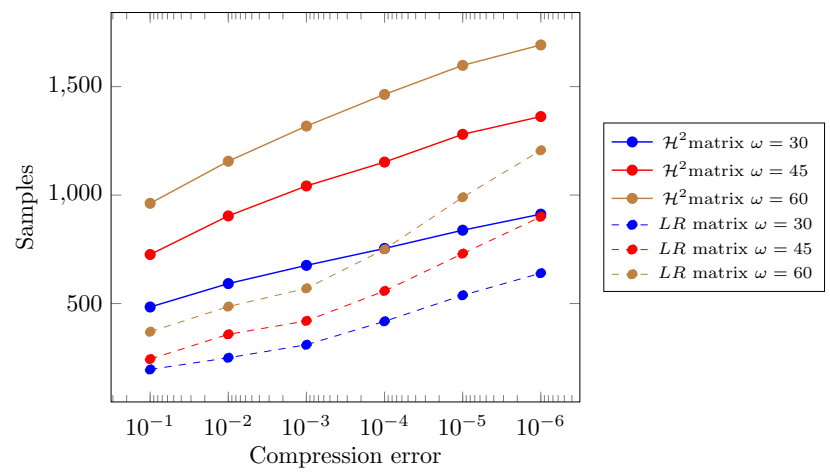

Fig. 4.7: Number of Hessian applications (samples) needed to construct the Gauss-Newton Hessians for different frequencies and different accuracies for hierarchical and global low rank approximations.

Figure 4.7 shows the number of samples needed to construct the Gauss-Newton Hessian evaluated at the converged parameter values for a range of frequencies and to accuracies in the range $10^{-6} \leq \epsilon \leq 10^{-1}$ measured in the relative 2-norm of the matrix. The sizes of the Hessians at the three frequencies $w=30,45$, and 60 are $n=8,211$, 18,164 , and 33,269 , respectively, growing with the square of the frequency to maintain about ten grid points per wave length. For a desired accuracy, the number of samples needed for the construction of the Hessian shows a slightly sublinear growth with frequency, and for a given frequency, a slow growth in the number of required samples with $|\log \epsilon|$. Due to the fairly limited information, coming from a single supersource input, the global low rank of the misfit Hessian requires fewer Hessian-vector products than the hierarchical representation. However as the accuracy increases the global 

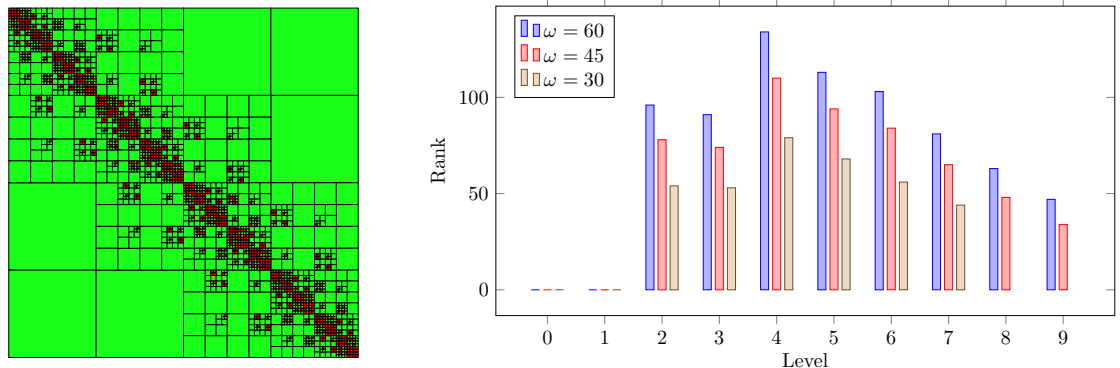

Fig. 4.8: Structure and max local ranks per level of Hessian at $\epsilon=10^{-4}$.

grows rapidly, particularly at higher frequencies. We expect that with more data incorporated in the inversion (using multiple independent sources, for example) the total number of required samples will grow slower for the hierarchical compression than for the global low rank approximation.

Figure 4.8 shows the structures and the local ranks of the resulting compressed Hessians. A leaf size of 64 was used in the construction with an admissibility condition that produces the structure shown. The matrix structure for the larger problems corresponding to $\omega=45,60$ is visually similar to the matrix structure of the smaller problem $\omega=30$ but is refined by two more levels. The resulting local ranks (maximum block rank) per level are shown for a relative accuracy of $\epsilon=10^{-4}$. We note the relatively mild sublinear growth in the local ranks with frequency.

4.4. Transient controlled-source electromagnetic inversion. In this section we consider a problem arising in transient controlled-source electromagnetism, see, e.g., $[73,36]$. The deterministic optimization approach to infer the spatially varying electrical conductivity field $\boldsymbol{\sigma}$ from observation data minimizes the least squares misfit between these observations and the response predicted by the model, i.e.,

$$
\underset{\boldsymbol{\sigma}: \Omega \rightarrow \mathbb{R}^{d \times d}}{\operatorname{minimize}} J(\boldsymbol{\sigma}):=\frac{1}{2} \sum_{r} \sum_{s} \int_{0}^{T}\left(\delta\left(\mathbf{x}_{r}\right) \mathbf{E}_{s}-\boldsymbol{d}_{r s}\right)^{2} d t+\alpha R(\boldsymbol{\sigma})=F(\boldsymbol{\sigma})+\alpha R(\boldsymbol{\sigma}) .
$$

Here, $\mathbf{E}_{s}$ is the electric vector field which is assumed to satisfy Maxwell's equations in the low frequency regime

$$
\begin{aligned}
\boldsymbol{\sigma} \partial_{t} \mathbf{E}_{s}+\nabla \times\left(\mu^{-1} \nabla \times \mathbf{E}_{s}\right) & =-\mathbf{J}_{s} & & \text { on } \Omega \times(0, T], \\
\mathbf{E}_{s} \times \mathbf{n} & =0 & & \text { on } \partial \Omega \times[0, T], \\
\mathbf{E}_{s} & =0 & & \text { on } \Omega \times\{0\},
\end{aligned}
$$

where $\mu>0$ is the constant corresponding to the magnetic permeability of free space, $\boldsymbol{d}_{r s}$ is the measured time-varying response at a receiver located at $\mathbf{x}_{r}$ corresponding to a given source term $\mathbf{J}_{s}$. In our study, we consider a horizontal electric dipole point source at $\mathbf{x}_{s}$ oriented along the $x$-axis direction, which leads to $\mathbf{J}_{s}=\delta\left(\mathbf{x}_{s}\right) \mathbf{e}_{\mathbf{1}} \partial_{t} W(t)$, where $W$ is a Ricker wavelet of the type [27]

$$
W(t):=\left(a-\frac{1}{2}\right) e^{-a}, \quad a=\left(\frac{\pi\left(t-t_{s}\right)}{t_{p}}\right)^{2}, \quad t_{s}=1.4 t_{p},
$$


and $\delta(\cdot)$ is the Dirac delta function. The continuous gradient of $F$ (we do not include the regularization in the expressions below) at a parameter $\boldsymbol{\sigma}_{0}$ may be computed as:

$$
\frac{\partial F}{\partial \boldsymbol{\sigma}}\left(\boldsymbol{\sigma}_{0}\right)=\sum_{s} \int_{0}^{T} \partial_{t} \mathbf{E}_{s} \cdot \mathbf{P}_{s} d t
$$

where $\mathbf{E}_{s}$ is given by solving the state equation (4.10) for the $s$-th source and with conductivity $\boldsymbol{\sigma}_{\mathbf{0}}$, and $\mathbf{P}_{s}$ is obtained by solving the adjoint PDE

$$
\begin{aligned}
-\boldsymbol{\sigma}_{0} \partial_{t} \mathbf{P}_{s}+\nabla \times\left(\mu^{-1} \nabla \times \mathbf{P}_{s}\right) & =-\sum_{r}\left(\delta\left(\mathbf{x}_{r}\right) \mathbf{E}_{\mathbf{s}}(t)-\boldsymbol{d}_{r s}(t)\right) & & \text { on } \Omega \times[0, T), \\
\mathbf{P}_{s} \times \mathbf{n} & =0 & & \text { on } \partial \Omega \times[0, T], \\
\mathbf{P}_{s} & =0 & & \text { on } \Omega \times\{T\} .
\end{aligned}
$$

Taking another variation, the product of the Hessian of $F$ at a parameter $\boldsymbol{\sigma}_{0}$, with a model perturbation $\boldsymbol{\nu}: \Omega \rightarrow \mathbb{R}^{d \times d}$ is computed as

$$
\frac{\partial^{2} F}{\partial \boldsymbol{\sigma}^{2}}\left(\boldsymbol{\sigma}_{0}\right) \boldsymbol{\nu}=\sum_{s} \int_{0}^{T} \partial_{t} \mathbf{E}_{s} \mathbf{Q}_{s} d t+\sum_{s} \int_{0}^{T} \partial_{t} \mathbf{F}_{s} \mathbf{P}_{s} d t,
$$

where $\mathbf{E}_{s}$ and $\mathbf{P}_{s}$ are given by solving (4.10) and (4.11), respectively, and $\mathbf{F}_{s}$ and $\mathbf{Q}_{s}$ are obtained from the solution of the incremental forward problem

$$
\begin{aligned}
\boldsymbol{\sigma}_{0} \partial_{t} \mathbf{F}_{s}+\nabla \times\left(\mu^{-1} \nabla \times \mathbf{F}_{s}\right) & =-\boldsymbol{\nu} \partial_{t} \mathbf{E}_{s} & & \text { on } \Omega \times(0, T], \\
\mathbf{F}_{s} \times \mathbf{n} & =0 & & \text { on } \partial \Omega \times[0, T], \\
\mathbf{F}_{s} & =0 & & \text { on } \Omega \times\{0\},
\end{aligned}
$$

and the incremental adjoint problem

$$
\begin{array}{rlrl}
-\boldsymbol{\sigma}_{0} \partial_{t} \mathbf{Q}_{s}+\nabla \times\left(\mu^{-1} \nabla \times \mathbf{Q}_{s}\right) & =-\sum_{r} \delta\left(\mathbf{x}_{r}\right) \mathbf{F}_{s}-\boldsymbol{\nu} \partial_{t} \mathbf{P}_{s} & & \text { on } \Omega \times[0, T), \\
\mathbf{Q}_{s} \times \mathbf{n}=0 & & \text { on } \partial \Omega \times[0, T], \\
\mathbf{Q}_{s} & =0 & & \text { on } \Omega \times\{T\} .
\end{array}
$$

The Hessian matrix-vector products are computed by using the software framework PETScOPT [68]. We discretize the state and ajoint variables with hexahedral Nedelec vector finite elements using the open-source library MFEM [6], which supports adaptive mesh refinement and higher-order elements. For the parameter space, we use standard Lagrange elements. For time integration of (4.10), (4.11), (4.13) and (4.14), we use the Crank-Nicholson scheme as implemented in the PETSc library [9]. For all the PDE considered, at each time step, we need to solve a poorly conditioned linear system with the matrices

$$
\frac{1}{\Delta t} M+A, \quad M_{i j}=\int_{\Omega} \phi_{j} \cdot \phi_{i} d \mathbf{x}, \quad A_{i j}=\int_{\Omega} \mu^{-1} \nabla \times \phi_{j} \cdot \nabla \times \phi_{i} d \mathbf{x},
$$

where $\phi_{i}$ is the $i$-th Nedelec finite element basis function for the Nedelec element space and $\Delta t$ the time step. These solves represents a key challenge for scalability. We use conjugate gradients preconditioned with the Balancing Domain Decomposition by Constraints solver [70, 71, 72] combined with a robust choice of the initial guess based on a reduced basis approximation scheme [69]. For the reported experiments, 

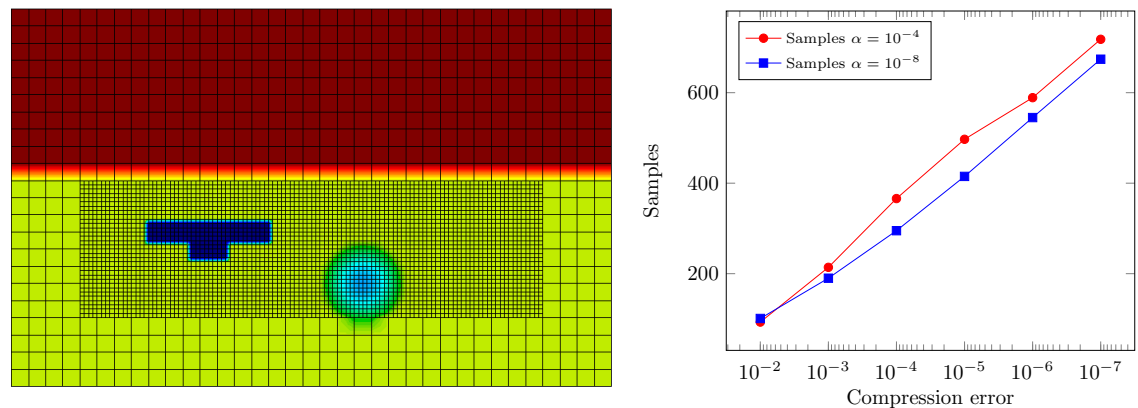

Fig. 4.9: Shown on the left is the truth parameter field to be recoverd from CSEM data, together with the locally refined discretization overlaid on material properties, which contain two anomalies (in blue). The right figure shows the total number of Hessian vector samples needed for hierarchical compression as a function of accuracy for different regularization parameters $\alpha$ of the TV regularizer.

this strategy leads, remarkably, to an average of less than one linear iterations per time step.

To illustrate the hierarchically low rank nature of the Hessians given in (4.9), we generate synthetic data for a two-dimensional isotropic tensor $\boldsymbol{\sigma}$ whose spatial distribution is given in Figure 4.9; the simulated time is 2 seconds, with a constant time step of 0.01 seconds. The values used for the conductivity field, reported in logarithmic scale in the figure, are $3.0 \mathrm{~S} / \mathrm{m}$ (water), $0.1 \mathrm{~S} / \mathrm{m}$ (sediments), $0.01 \mathrm{~S} / \mathrm{m}$ (salt), and $0.001 \mathrm{~S} / \mathrm{m}$ for the T-shaped anomaly region. The non-conforming mesh used to discretize the conductivity is also shown. The setup uses an array of 14 equispaced sources located at the water/sediments interface and an array of 18 receivers placed right in between the water/sediments interface and the non-conforming mesh interface. The region of interest for the optimization process extends to the first layer of elements surrounding the non-conforming interface, for a total of 2381 optimization degrees of freedom. For the optimization, we used a primal-dual total variation regularizer [28], which preserves sharp gradients in the coefficient values close to the data recording location, and guarantees an almost optimal line-search process.

Results for Hessian compressibility reported here consider the full Hessian as given in (4.12). Figure 4.9 shows the number of samples needed to construct the Hessian at convergence of the inversion process. We note two characteristics of hierarchical representations in this numerical experiment. The first is the log dependence of the number of samples (and local ranks, not shown here) on the desired accuracy. The second is the relative insensitivity of the local ranks to the amount regularization. The global rank of the matrix does not have a direct effect on the local ranks.

Figure 4.10 shows the structure of the hierarchical low rank Hessian approximation. Note the importance of ordering the matrix in a spatially-aware manner so that indices that are close correspond to nodes that are in spatial proximity. The figure depicts the entries of the Hessian when the nodes are ordered lexicographically and re-ordered using a KD-tree binary spatial partitioning. The reordering of the optimization degrees of freedom, results in larger blocks that are "smooth", a necessary characteristic for an efficient hierarchically low rank representation.

5. Conclusions. In this paper we presented a hierarchical matrix representation of Hessians as a viable and practical representation for storing and manipulating 

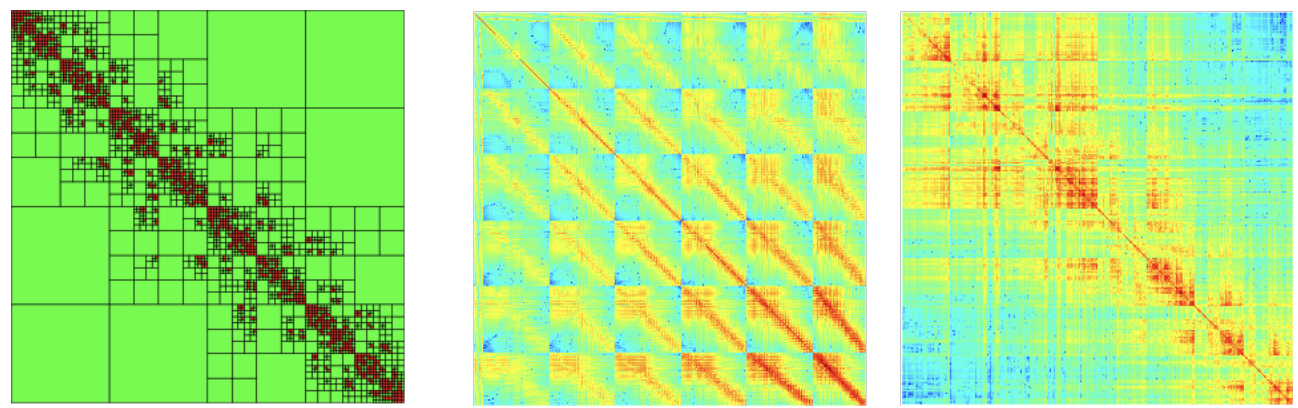

Fig. 4.10: Shown in the left figure is the structure of the hierarchical Hessian using the KD-tree ordering. Middle and right figures show the Hessian entries with lexicographic and KD-tree ordering of mesh nodes, respectively. Color reflect magnitude of absolute value, red is largest and blue is smallest.

second order information for inverse problems governed by PDEs, which we expect will be applicable to a broader class of PDE-constrained optimization problems. We have shown the increasing superiority of hierarchical matrix approximation of the data misfit Hessian over a low rank approximation as the data become more informative for four inverse problems governed by diffusion, transport, and acoustic and electromagnetic wave propagation. Since the eigenvalues of the data misfit Hessian typically decay more slowly in higher dimensional problems, we expect the hierarchical approximations to continue to be effective for inverse problems in three dimensions, and plan to study their performance in future work.

The primary advantages of the hierarchical representation are that it provides a tunable accuracy approximation, it has a small and asymptotically optimal memory footprint, and it may be generated from Hessian-vector products that are generally available via state and adjoint PDE solves. Our numerical results have shown that the Hessians that appear in applications do, as predicted, admit a hierarchical structure and can therefore be represented with blocks at different levels of granularity that have small bounded rank. Once the hierarchical Hessian approximation is computed, it can also be operated on very efficiently, purely algebraically, for performing operations such matrix-vector multiplications, local and global low rank updates, inversion, and square roots.

The machinery of hierarchical Hessians is general, algebraic, and effective in storing and manipulating the formally dense Hessians arising in a variety of PDE-governed inverse problems. Our initial results on moderately-sized problems are encouraging, and we intend to study the computational advantages and effectiveness of hierarchical Hessians at large scale in future work.

\section{Appendix A. Iterative Methods for Inverse Hessian Approximation.}

Iterative methods for computing the matrix inverse have a long history dating back to [59]. Under reasonably general assumptions, they can be shown to be globally convergent methods, and require $2 \log \kappa+\log \log (1 / \epsilon)$ number of iterations to converge to an $\epsilon$ accuracy, where $\kappa$ is the condition number of the matrix being inverted [60].

Iterative inversion methods have not been very popular (but see [58]) because at face value their computational cost is larger than direct factorization-based inversion methods as they require two $O\left(n^{3}\right)$ matrix multiplications per iteration when used with dense storage. This calculus changes with hierarchical matrices, as the product 
of two such matrices may be obtained in $\log$-linear time $O(k n \log n)$ [20]. This fast matrix multiply makes iterative inversion methods attractive here, even with memory considerations aside.

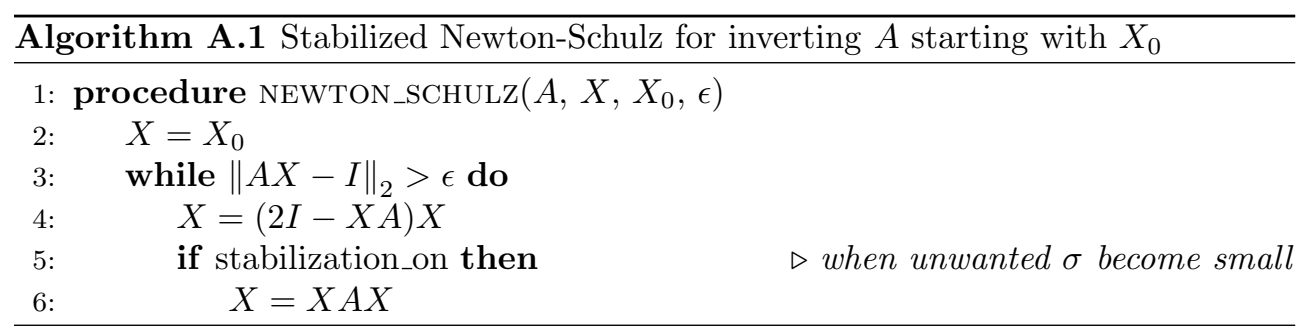

A basic stabilized Newton-Schulz (NS) iteration shown in Algorithm A.1. For symmetric positive-definite matrices, a scaled identity $X_{0}=I /\|A\|_{\infty}$ may be used as the starting iterate and results in globally convergent iterations. Newton-Schulz converges only linearly in the early iterations before entering the rapid quadratic convergence regime. Its convergence can be sped up with improved initial guesses and convergence accelerations [54]. In the current optimization context, the inverse from a previous iterate provides a natural starting point and this warm-start reduces the number of iterations compared to an agnostic starting point, as we illustrate below. The basic NS iteration is numerically stable and even self-correcting for nonsingular matrices. It may also be used to compute the Moore-Penrose pseudoinverse for singular matrices. However, for such matrices, and for matrices $A(\epsilon)$ where singular values below $\epsilon$ are ignored, it may be mildly unstable. The correction step in lines 5-6 of Algorithm A.1 insures stability and need only be activated after the unwanted singular values of $X A$ are small enough, a condition that can be inexpensively monitored [54].

A.1. Newton-Schulz iterations with hierarchical matrices. To adapt iterative methods to the hierarchical matrix representation context, two modifications are needed as shown in Algorithm A.2. The first comes from the fact that the primary fast operation we have, especially on GPUs, is a blocked matrix-vector multiplication; the construction of the Schulz iterates is done via the procedure of subsection 3.3 where a sampler, i.e., a matrix-vector expression evaluator, is provided to the construction procedure at every iteration. For example, a simple sampler for NS would follow Algorithm A.3 to produce the samples $Y$ from random input vectors.

The second, more consequential modification, is that the iterative algorithm may be carried out with truncation - an option made possible by the tunable-accuracy nature of the hierarchical representation. As solving exactly the tangent system in the early phases of the Newton method is a waste of computational resources, it makes little sense to construct the matrices in the first iterations to the ultimately desired accuracy. In fact, Hackbusch et al. [38] showed that, under fairly general conditions, the intermediate iterates $X_{k}$ may be replaced by approximations without affecting the convergence rate of the method. Therefore we have the freedom to choose the accuracy $\epsilon_{k}$ to which the intermediate iterates are to be generated. A more effective strategy starts with a low accuracy in the early iterations and gradually reduces it as convergence is approached. As we show below, the use of such a dynamic threshold reduces substantially the overall computational cost of inversion by producing intermediate iterates with smaller footprints.

We show the effectiveness of Algorithm A.2 on the following minimal surface 

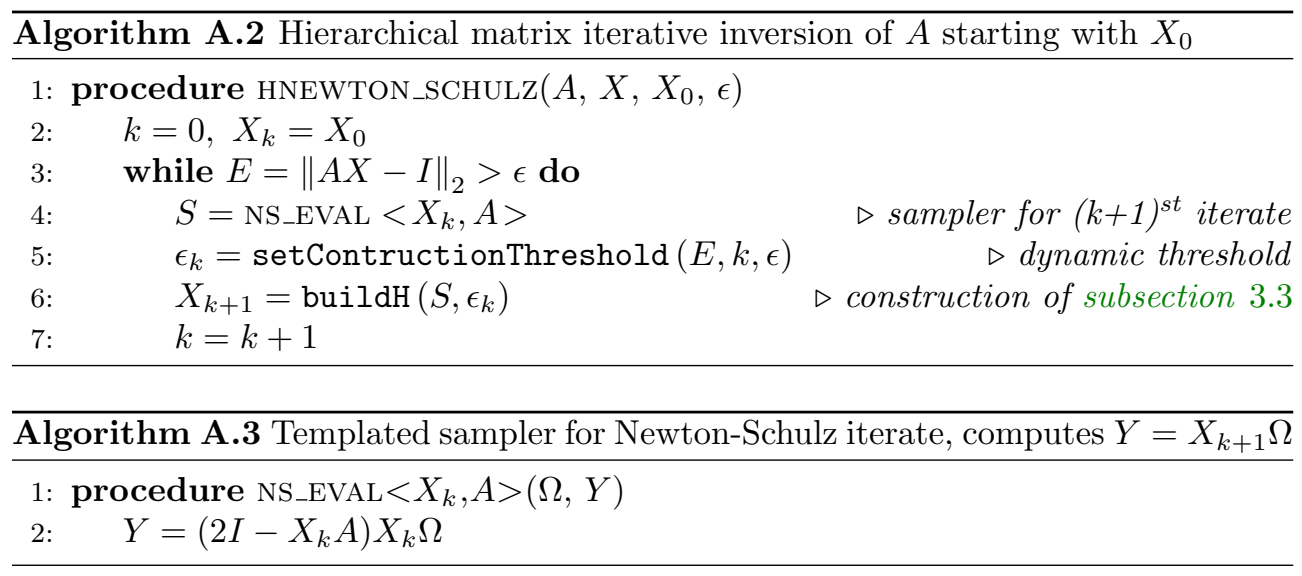

problem. Let $m(x)$ be the height of a surface defined in the unit square $\Omega=[0,1]^{2}$.

$$
\begin{array}{ll}
\underset{m(x)}{\operatorname{minimize}} & J(m)=\int_{\Omega} \sqrt{1+|\nabla m|^{2}} d \Omega, \\
\text { subject to } & m(\partial \Omega)=m_{0} .
\end{array}
$$

We discretize $m(x)$ and the objective using finite differences on an $n=128 \times 128$ grid. The Hessian for this problem can be computed exactly, up to discretization errors, since it corresponds to the linearization of a nonlinear Poisson equation. Its inverse, however, is dense, and therefore it is a good test for the effectiveness of the iterative inversion method described above, since all resulting approximation errors are attributable to it.

For the following experiments, we set the compression threshold used during the construction of the hierarchical matrix to $\epsilon_{k}=10^{-6}$ with a leaf size of 64 . Starting from the scaled identity $X_{0}=\frac{I}{\|A\|_{\infty}}$, inverting the Hessian using NS for the first optimization step needs quite a few iterations, with the number of required samples for intermediate iterates increasing very rapidly before receding as it converges as shown in Figure A.1a. The total time needed to invert the Hessian using this method is about 178 seconds on a P100 GPU, with over $75 \%$ of the total runtime spent in compression. To alleviate the impact of the intermediate iterates, we can start with a relatively loose compression error threshold $\epsilon_{k}$, tightening the threshold as we converge. This significantly reduces the number of samples needed for the earlier iterations as shown in Figure A.1a, where we start with a much looser threshold of $10^{-2}$. The total runtime is then reduced to $51 \mathrm{~s}$, a $3.5 \times$ reduction in inversion time, with $69 \%$ of the time spent in compression.

From the second optimization iteration onwards, we can use the approximate Hessian inverse from the previous iteration as an initial guess, allowing NS to converge in fewer iterations. While the first optimization iteration converged in 16 NS iterations, the second iteration needed only 6 , inverting the Hessian in 32 s if a static threshold is used and in 14s with a dynamic threshold. In Figure A.1b, we compare the number of needed samples for static and dynamic thresholds. Finally, the error in the inverse $\|A X-I\|_{2}$ throughout the NS procedure is shown in Figure A.1c for a given optimization step, exhibiting rapid convergence as we approach the solution. Warm-starting from previous iterations results in the iterations entering the fast convergence regime earlier. 


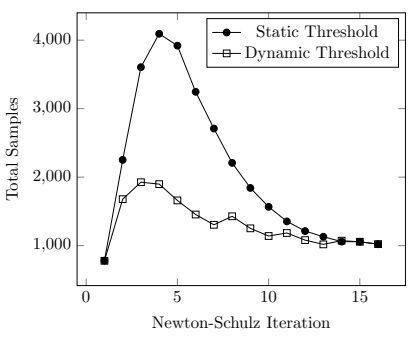

(a)

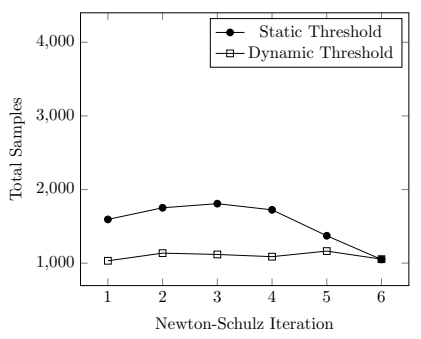

(b)

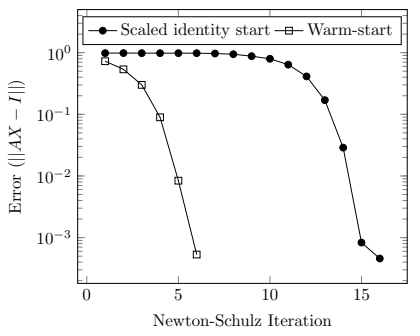

(c)

Fig. A.1: Performance of Hessian inversion on a problem of size $n=16,384$. Number of Hessian-vector products throughout the NS iterations: (a) starting from a scaled identity and (b) a warm starting from previous iteration. (c) Convergence history.

A.2. Higher order methods for faster convergence. The results above show that the hierarchical matrix compression portion of the construction algorithm dominates the total runtime of the inversion algorithm. This is because we apply the low rank updates generated during the sampling phase in relatively small blocks due to memory constraints of the GPU. The majority of the resulting linear algebra operations during compression, such as batched rank-revealing QR and tall skinny QR decompositions, are not particularly efficient on GPUs and are relatively costly. On the other hand, the sampling operations, which primarily consist of batched matrix-matrix products and blocked sparse matrix vector products, are highly parallel, efficient, and arithmetically intensive. Taking this disparity into consideration, we employ high order hyperpower iterative methods to shift the computational load to the sampling phase. An order $l$ hyperpower iteration is defined as [55]

$$
X_{k+1}=X_{k}\left(I+R_{k}+\cdots+R_{k}^{l-1}\right)=X_{k} \sum_{i=0}^{l-1} R_{k}^{i}
$$

where $R_{k}=I-A X_{k}$, involving $l$ matrix products. Setting $l=2$ gives us the standard NS iteration of the previous section. Most previous work on efficient hyperpower iterations with dense matrices attempt to reduce the number of matrix-matrix products by calculating a few temporary matrices and factoring the summation in (A.2). Here, we seek to avoid the costly compression for those temporary matrices, and use the original form of the equation which performs $l$ products. This achieves our goal of concentrating the workload on the far more efficient sampling phase of the computation, and achieves considerable time savings. Sampling $X_{k+1}$ can be done efficiently using a method similar to Horner's method for polynomial evaluation, as shown in Algorithm A.4 which replaces the NS evaluation of line 4 of Algorithm A.2.

The performance of higher order methods is illustrated in Figure A.2a which shows the number of iterations and the samples taken in each iteration for hyperpower iterations of order $l=8,16,32$, which are notably lower than those of Figure A.1a. High order methods also have the benefit of faster convergence as shown in Figure A.2b where the order 8 method takes 7 iterations to converge and the order 16 and 32 methods take 6 iterations, as opposed to the 16 iterations needed by the second order method. The overall inversion times are also considerably lower, with order 8 and 16 at 20 seconds and the order 32 at $25 \mathrm{~s}$. The order 8 method does one more iteration than the order 16 method, but the lower sampling cost puts it on equal footing, 


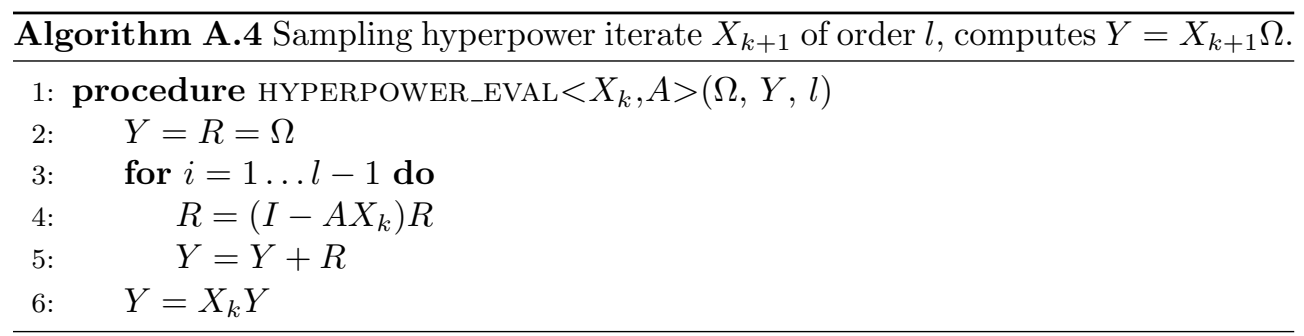

whereas the order 32 performs the same number of iterations as the order 16 method while having higher cost per sample.

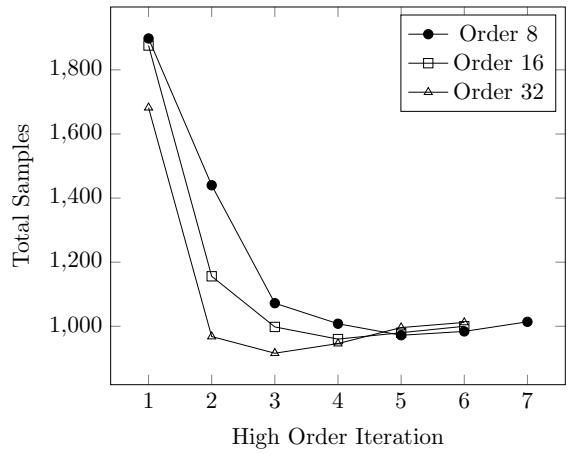

(a) Number of samples vs iterations.

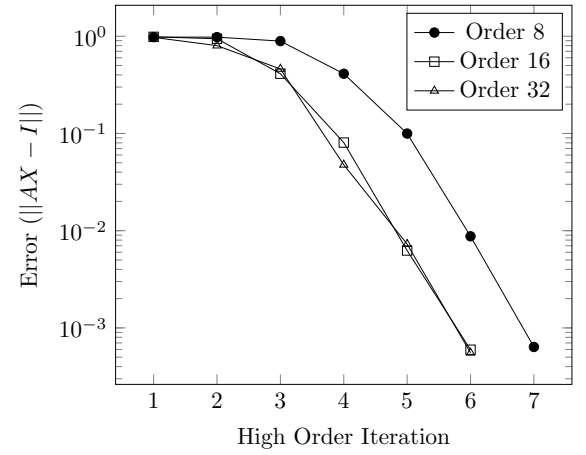

(b) Convergence history.

Fig. A.2: Performance of Hessian inversion using high order iterations of order 8, 16, and 32 .

A.3. Unrolling iterations. As another algorithmic optimization for the example problem (A.1), we consider the effect of loop unrolling. The low number of NS iterations needed for the second optimization iteration onwards, due to the use of the previous approximate Hessian inverse as the initial NS iterate, presents another opportunity for greater inversion performance. By unrolling the iterates to express $X_{k}$ in terms of $X_{0}$, we can achieve the same goal as the higher order methods, where the workload is shifted to the sampling phase and the increase in samples for the intermediate iterates can be avoided; the $k$-th unrolled iterate can be evaluated as the hyperpower iterates as:

$$
\begin{aligned}
X_{k} & =X_{k-1}\left(2 I+A X_{k-1}\right) \\
& =X_{k-2}\left(2 I+A X_{k-2}\right)\left(2 I+A X_{k-2}\left(2 I+A X_{k-2}\right)\right)=\ldots \\
& =X_{0} \sum_{i=0}^{2^{k}}\left(\begin{array}{c}
2^{k} \\
i
\end{array}\right)\left(-A X_{0}\right)^{i} .
\end{aligned}
$$

Since the number of entries in the sum grows exponentially, we can efficiently unroll only a small number of iterations (say 5 or 6 ); however, since the number of iterations needed after the first optimization iteration is small, we can effectively unroll all of the required iterations, and obtain the approximate inverse in a single construction. This further reduces the inversion time from 14 seconds to 4 seconds, giving us another $3.5 \times$ improvement on the dynamic error threshold inversion. 
We remark that the inversion methods introduced in this section demonstrate the performance benefits of concentrating the computational effort in the arithmetically intensive sampling phase, not only in terms of reducing inversion time as in the unrolled iterations, but also in terms of reducing the total number of iterations required for convergence, as in the higher order methods. Related methods for computing square roots and inverse square roots may be similarly formulated and optimized.

Acknowledgments. This work was supported by the King Abdullah University of Science and Technology (KAUST) Office of Sponsored Research (OSR) under Award No: OSR-2018-CARF-3666.

\section{REFERENCES}

[1] A. Alexanderian, P. J. Gloor, and O. Ghattas, On Bayesian A-and D-optimal experimental designs in infinite dimensions, Bayesian Analysis, 11 (2016), pp. 671-695, https://doi.org/10.1214/15-BA969. arXiv preprint arXiv:1408.6323.

[2] N. Alger, Data-Scalable Hessian Preconditioning for Distributed Parameter PDE-Constrained Inverse Problems, PhD thesis, The University of Texas at Austin, 2019.

[3] N. Alger, V. Rao, A. Meyers, T. Bui-Thanh, and O. Ghattas, Scalable matrix-free adaptive product-convolution approximation for locally translation-invariant operators, SIAM Journal on Scientific Computing, (2019). To appear.

[4] N. Alger, U. Villa, T. Bui-Thanh, and O. Ghattas, A data scalable augmented Lagrangian KKT preconditioner for large scale inverse problems, SIAM Journal on Scientific Computing, 39 (2017), pp. A2365-A2393, https://doi.org/10.1137/16M1084365.

[5] S. Ambikasaran and E. Darve, $A n \mathcal{O}(N \log N)$ fast direct solver for partial Hierarchically Semiseparable matrices, Journal of Scientific Computing, 57 (2013), pp. 477-501.

[6] R. Anderson, A. Barker, J. Bramwell, J. Cerveny, J. Dahm, V. Dobrev, Y. Dudouit, A. Fisher, T. Kolev, M. Stowell, V. Tomov, And S. Zampini, MFEM: A modular finite element methods library, In preparation, (2019).

[7] D. N. Arnold, F. Brezzi, B. Cockburn, And L. D. Marini, Unified analysis of discontinuous Galerkin methods for elliptic problems, SIAM Journal on Numerical Analysis, 39 (2002), pp. 1749-1779.

[8] B. Ayuso and L. D. Marini, Discontinuous galerkin methods for advection-diffusion-reaction problems, SIAM Journal on Numerical Analysis, 47 (2009), pp. 1391-1420.

[9] S. BALAY ET AL., PETSc users manual: Revision 3.10, tech. report, Argonne National Lab.(ANL), Argonne, IL (United States), 2018.

[10] O. Bashir, K. Willcox, O. Ghattas, B. van Bloemen Waanders, and J. Hill, Hessianbased model reduction for large-scale systems with initial condition inputs, International Journal for Numerical Methods in Engineering, 73 (2008), pp. 844-868.

[11] M. BeBENDORF, Low-rank approximation of elliptic boundary value problems with high-contrast coefficients, SIAM Journal on Mathematical Analysis, 48 (2016), pp. 932-949.

[12] M. Bebendorf and W. HackBusch, Existence of $\mathcal{H}$-matrix approximants to the inverse FE-matrix of elliptic operators with $L^{\infty}$-coefficients, Numerische Mathematik, 95 (2003), pp. $1-28$.

[13] J.-P. Berenger, A perfectly matched layer for the absorption of electromagnetic waves, Journal of computational physics, 114 (1994), pp. 185-200.

[14] A. Beskos, M. Girolami, S. Lan, P. E. Farrell, and A. M. Stuart, Geometric MCMC for infinite-dimensional inverse problems, Journal of Computational Physics, 335 (2017), pp. 327-351.

[15] S. Börm, Data-sparse approximation of non-local operators by $\mathcal{H}^{2}$-matrices, Linear Algebra and its Applications, 422 (2007), pp. $380-403$.

[16] S. Börm, Approximation of solution operators of elliptic partial differential equations by $\mathcal{H}$ and $\mathcal{H}^{2}$-matrices, Numerische Mathematik, 115 (2010), pp. 165-193.

[17] S. BöRm, Directional $\mathcal{H}^{2}$-matrix compression for high-frequency problems, Numerical Linear Algebra with Applications, 24 (2017), p. e2112.

[18] A. Borzì And V. Schulz, Computational Optimization of Systems Governed by Partial Differential Equations, SIAM, 2012.

[19] W. Boukaram, G. Turkiyyah, and D. Keyes, Hierarchical matrix operations on GPUs: Matrix-vector multiplication and compression, ACM Transactions on Mathematical Software, 45 (2019), pp. 3:1-3:28. 
[20] W. Boukaram, G. Turkiyyah, and D. Keyes, Randomized GPU algorithms for the construction of hierarchical matrices from mat-vec operations, SIAM Journal on Scientific Computing, (in press) (2019).

[21] T. Bui-Thanh, C. Burstedde, O. Ghattas, J. Martin, G. Stadler, and L. C. Wilcox, Extreme-scale UQ for Bayesian inverse problems governed by PDEs, in SC12: Proceedings of the International Conference for High Performance Computing, Networking, Storage and Analysis, 2012.

[22] T. Bui-Thanh and O. Ghattas, Analysis of the Hessian for inverse scattering problems. Part I: Inverse shape scattering of acoustic waves, Inverse Problems, 28 (2012), p. 055001, https://doi.org/10.1088/0266-5611/28/5/055001.

[23] T. Bui-Thanh and O. Ghattas, Analysis of the Hessian for inverse scattering problems. Part II: Inverse medium scattering of acoustic waves, Inverse Problems, 28 (2012), p. 055002, https://doi.org/10.1088/0266-5611/28/5/055002.

[24] T. Bui-Thanh and O. Ghattas, Analysis of the Hessian for inverse scattering problems. Part III: Inverse medium scattering of electromagnetic waves, Inverse Problems and Imaging, 7 (2013), pp. 1139-1155.

[25] T. Bui-Thanh, O. Ghattas, J. Martin, and G. Stadler, A computational framework for infinite-dimensional Bayesian inverse problems Part I: The linearized case, with application to global seismic inversion, SIAM Journal on Scientific Computing, 35 (2013), pp. A2494-A2523, https://doi.org/10.1137/12089586X.

[26] T. Bui-Thanh and M. A. Girolami, Solving large-scale PDE-constrained Bayesian inverse problems with Riemann manifold Hamiltonian Monte Carlo, Inverse Problems, 30 (2014), p. 114014.

[27] J. M. CARCione, Simulation of electromagnetic diffusion in anisotropic media, Progress In Electromagnetics Research, 26 (2010), pp. 425-450.

[28] T. F. Chan, G. H. Golub, And P. Mulet, A nonlinear primal-dual method for total variationbased image restoration, SIAM Journal on Scientific Computing, 20 (1999), pp. 1964-1977, https://doi.org/10.1137/S1064827596299767.

[29] B. Crestel, G. Stadler, and O. Ghattas, A comparative study of structural similarity and regularization for joint inverse problems governed by PDEs, Inverse Problems, 35 (2018), p. 024003, https://doi.org/10.1088/1361-6420/aaf129.

[30] T. Cui, K. Law, and Y. Marzouk, Dimension-independent likelihood-informed MCMC, Journal of Computational Physics, 304 (2016), pp. 109-137.

[31] L. Demanet, P.-D. Létourneau, N. Boumal, H. Calandra, J. Chiu, and S. Snelson, Matrix probing: a randomized preconditioner for the wave-equation Hessian, Applied and Computational Harmonic Analysis, 32 (2012), pp. 155-168.

[32] S. C. Eisenstat And H. F. Walker, Choosing the forcing terms in an inexact Newton method, SIAM Journal on Scientific Computing, 17 (1996), pp. 16-32.

[33] I. Epanomeritakis, V. Akçelik, O. Ghattas, and J. Bielak, A Newton-CG method for large-scale three-dimensional elastic full-waveform seismic inversion, Inverse Problems, 24 (2008), p. 034015 (26pp), https://doi.org/10.1088/0266-5611/24/3/034015.

[34] P. H. Flath, L. C. Wilcox, V. Akçelik, J. Hill, B. van Bloemen Waanders, and O. GhatTAS, Fast algorithms for Bayesian uncertainty quantification in large-scale linear inverse problems based on low-rank partial Hessian approximations, SIAM Journal on Scientific Computing, 33 (2011), pp. 407-432, https://doi.org/10.1137/090780717.

[35] L. Grasedyck and W. Hackbusch, Construction and arithmetics of $\mathcal{H}$-matrices, Computing, 70 (2003), pp. 295-334.

[36] E. HABER, Computational Methods in Geophysical Electromagnetics, Mathematics in Industry, Society for Industrial and Applied Mathematics, 2014.

[37] W. Hackbusch, Hierarchical Matrices: Algorithms and Analysis, Springer, 2015.

[38] W. Hackbusch, B. N. Khoromskij, and E. E. Tyrtyshnikov, Approximate iterations for structured matrices, Numerische Mathematik, 109 (2008), pp. 365-383.

[39] N. Halko, P. Martinsson, And J. A. Tropp, Finding structure with randomness: Probabilistic algorithms for constructing approximate matrix decompositions, SIAM Review, 53 (2011), pp. 217-288.

[40] M. Heinkenschloss, Mesh independence for nonlinear least squares problems with norm constraints, SIAM Journal on Optimization, 3 (1993), pp. 81-117.

[41] M. Hesse AND G. StadLer, Joint inversion in coupled quasistatic poroelasticity, Journal of Geophysical Research: Solid Earth, 119 (2014), pp. 1425-1445.

[42] N. J. Higham, Accuracy and stability of numerical algorithms, vol. 80, SIAM, 2002.

[43] M. Hinze, R. Pinnau, M. Ulbrich, and S. Ulbrich, Optimization with PDE Constraints, Springer, 2009. 
[44] T. Isaac, N. Petra, G. Stadler, and O. Ghattas, Scalable and efficient algorithms for the propagation of uncertainty from data through inference to prediction for large-scale problems, with application to flow of the Antarctic ice sheet, Journal of Computational Physics, 296 (2015), pp. 348-368, https://doi.org/10.1016/j.jcp.2015.04.047.

[45] T. Kolda And B. BADER, Tensor decompositions and applications, SIAM Review, 51 (2009), pp. $455-500$.

[46] L. Lin, J. Lu, AND L. YING, Fast construction of hierarchical matrix representation from matrix-vector multiplication, Journal of Computational Physics, 230 (2011), pp. 40714087.

[47] A. LogG, K.-A. Mardal, G. N. Wells, et Al., Automated Solution of Differential Equations by the Finite Element Method, Springer, 2012, https://doi.org/10.1007/978-3-642-23099-8.

[48] J. Martin, L. C. Wilcox, C. Burstedde, and O. Ghattas, A stochastic Newton MCMC method for large-scale statistical inverse problems with application to seismic inversion, SIAM Journal on Scientific Computing, 34 (2012), pp. A1460-A1487.

[49] P. Martinsson, A fast randomized algorithm for computing a hierarchically semiseparable representation of a matrix, SIAM Journal on Matrix Analysis and Applications, 32 (2011), pp. 1251-1274.

[50] P. MARTinsson, Compressing rank-structured matrices via randomized sampling, SIAM Journal on Scientific Computing, 38 (2016), pp. A1959-A1986.

[51] R. Nammour, Approximate Multi-Parameter Inverse Scattering Using Pseudodifferential Scaling, PhD thesis, Rice University, 2011.

[52] R. Nammour and W. W. Symes, Approximate constant density acoustic inverse scattering using dip-dependent scaling, in SEG Technical Program Expanded Abstracts 2009, 2009, pp. 2347-2351.

[53] J. Nocedal and S. J. Wright, Numerical Optimization, Springer Verlag, Berlin, Heidelberg, New York, second ed., 2006.

[54] V. Pan And R. Schreiber, An improved Newton iteration for the generalized inverse of a matrix, with applications, SIAM J. Scientific Computing, 12 (1991), pp. 1109-1130.

[55] V. Pan, F. Soleymani, and L. ZhaO, An efficient computation of generalized inverse of a matrix, Applied Mathematics and Computation, 316 (2018), pp. 89 - 101.

[56] N. Petra, J. Martin, G. Stadler, and O. Ghattas, A computational framework for infinitedimensional Bayesian inverse problems: Part II. Stochastic Newton MCMC with application to ice sheet inverse problems, SIAM Journal on Scientific Computing, 36 (2014), pp. A1525-A1555.

[57] B. RIVIERE, Discontinuous Galerkin methods for solving elliptic and parabolic equations: theory and implementation, SIAM, 2008.

[58] P. SAnders, J. Speck, And R. StefFen, Work-efficient matrix inversion in polylogarithmic time, ACM Trans. Parallel Comput., 2 (2015), pp. 15:1-15:29.

[59] G. Schulz, Iterative Berechung der reziproken Matrix, ZAMM - Journal of Applied Mathematics and Mechanics / Zeitschrift für Angewandte Mathematik und Mechanik, 13 (1933), pp. $57-59$.

[60] T. SÖDERSTRÖM AND G. StewART, On the numerical properties of an iterative method for computing the moore-penrose generalized inverse, SIAM Journal on Numerical Analysis, 11 (1974), pp. 61-74.

[61] R. Versteeg, The marmousi experience: Velocity model determination on a synthetic complex data set, The Leading Edge, 13 (1994), pp. 927-936.

[62] U. Villa, N. Petra, and O. Ghattas, hIPPYlib: an Extensible Software Framework for Large-scale Deterministic and Bayesian Inverse Problems, Journal of Open Source Software, 3 (2018), https://doi.org/10.21105/joss.00940.

[63] J. Xia, S. Chandrasekaran, M. Gu, And X. S. Li, Fast algorithms for hierarchically semiseparable matrices, Numerical Linear Algebra with Applications, 17 (2010), pp. 953-976.

[64] Y. YAng AND B. ENGQUist, Analysis of optimal transport and related misfit functions in fullwaveform inversion, Geophysics, 83 (2018), pp. A7-A12.

[65] R. Yokota, G. Turkiyyah, And D. Keyes, Communication Complexity of the Fast Multipole Method and its Algebraic Variants, Supercomputing Frontiers and Innovations, 1 (2014), pp. 63-84.

[66] C. D. Yu, J. Levitt, S. Reiz, And G. Biros, Geometry-oblivious FMM for compressing dense SPD matrices, in Proceedings of the International Conference for High Performance Computing, Networking, Storage and Analysis, SC '17, 2017, pp. 53:1-53:14.

[67] C. D. YU, S. ReIZ, AND G. BIRos, Distributed-memory hierarchical compression of dense SPD matrices, in Proceedings of the International Conference for High Performance Computing, Networking, Storage, and Analysis, SC '18, 2018, pp. 15:1-15:15. 
[68] S. ZAMPInI, PETScOPT: A framework for high performance PDE-constrained optimization with PETSc. https://github.com/stefanozampini/petscopt/.

[69] S. ZAmPINI, Non-overlapping domain decomposition methods for three-dimensional cardiac reaction-diffusion models and applications, 2011.

[70] S. ZAMPINI, PCBDDC: a class of robust dual-primal methods in PETSc, SIAM Journal on Scientific Computing, 38 (2016), pp. S282-S306.

[71] S. Zampini, Adaptive BDDC deluxe methods for $H$ (curl), in Domain Decomposition Methods in Science and Engineering XXIII, Springer, Cham, 2017, pp. 285-292.

[72] S. Zampini, P. Vassilevski, V. Dobrev, and T. Kolev, Balancing domain decomposition by constraints algorithms for curl-conforming spaces of arbitrary order, in International Conference on Domain Decomposition Methods, Springer, Cham, 2017, pp. 103-116.

[73] M. Zaslavsky, V. Druskin, A. Abubakar, T. Habashy, and V. Simoncini, Large-scale Gauss-Newton inversion of transient controlled-source electromagnetic measurement data using the model reduction framework, Geophysics, 78 (2013), pp. E161-E171.

[74] H. Zhu, S. Li, S. Fomel, G. Stadler, and O. Ghattas, A Bayesian approach to estimate uncertainty for full waveform inversion with a priori information from depth migration, Geophysics, 81 (2016), pp. R307-R323. 